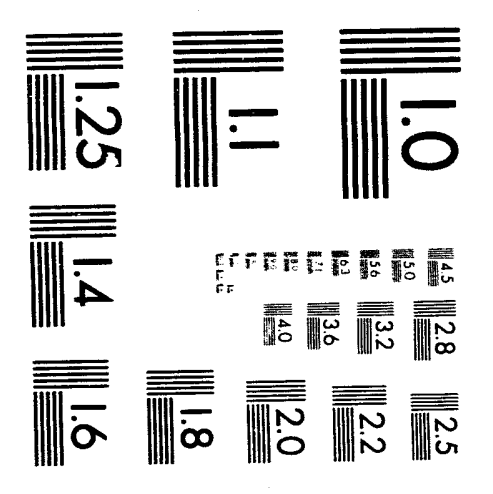



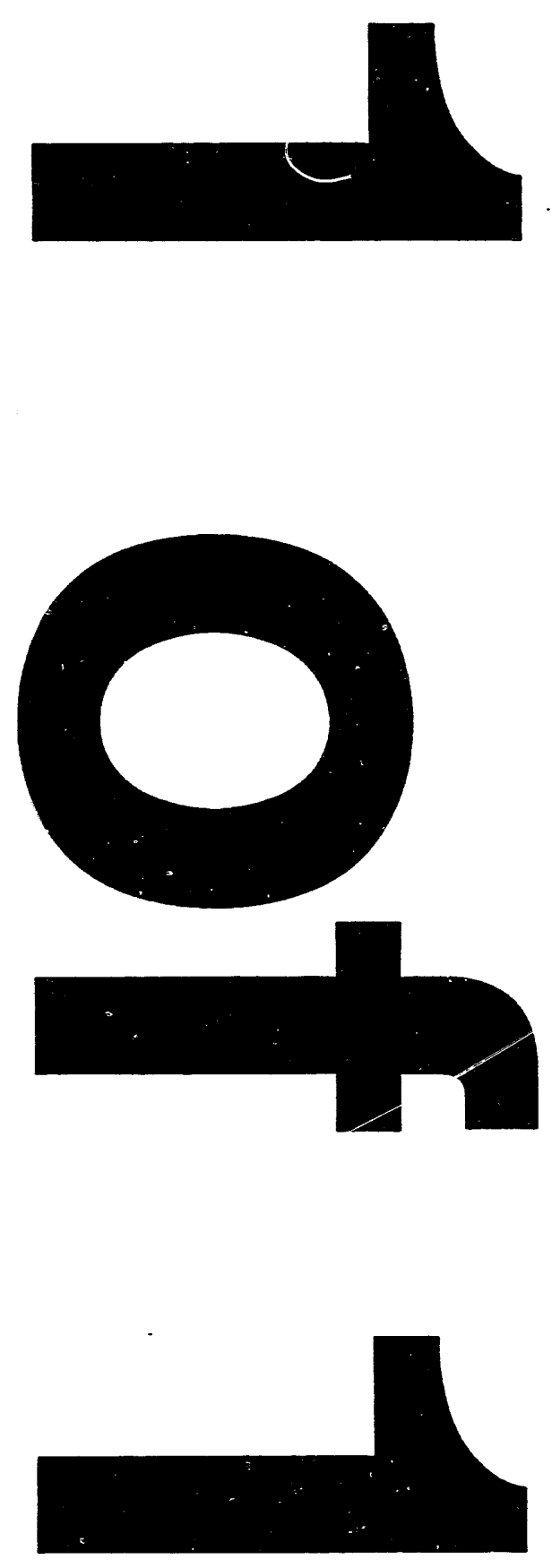


\title{
REENTRY VEHICLE ADAPTIVE TELEMETRY
}

\author{
R. E. Kidner \\ Telemetry Technology Development Department \\ Sandia National Laboratories \\ Albuquerque, NM 87185
}

\begin{abstract}
In RF telemetry (TM) the allowable RF bandwidth limits the amount of data in the telemetered data set. Typically the data set is less than ideal to accommodate all aspects of a test. In the case of diagnostic data, the compromise often leaves insufficient diagnostic data when problems occur. As a solution, intelligence was designed into a TM, allowing it to adapt to changing data requirements. To minimize the computational requirements for an intelligent TM, a fuzzy logic inference engine was developed. This inference engine was simulated on a PC and then loaded into a TM hardware package for final testing.
\end{abstract}

\section{MASTER}

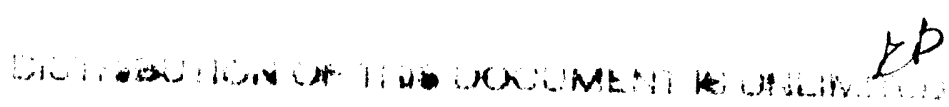




\section{CONTENTS}

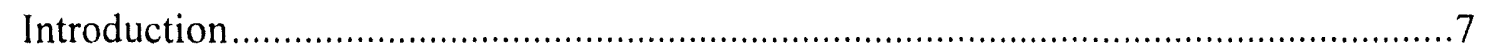

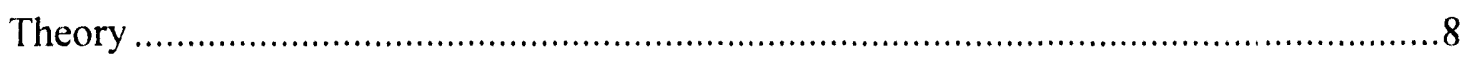

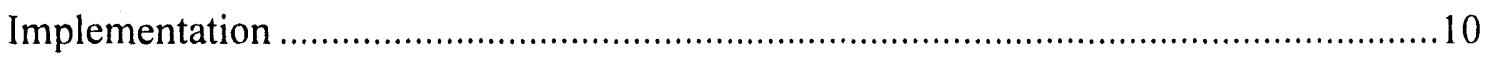

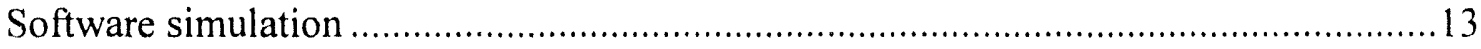

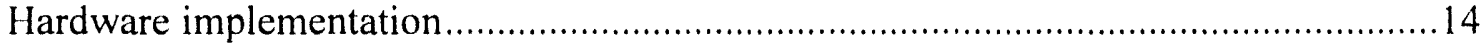

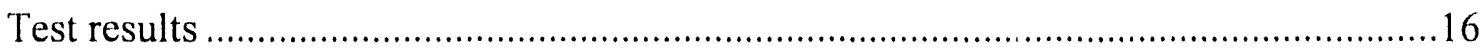

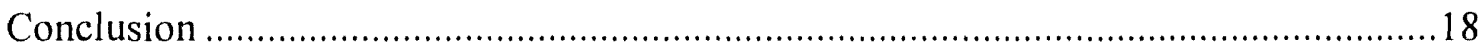

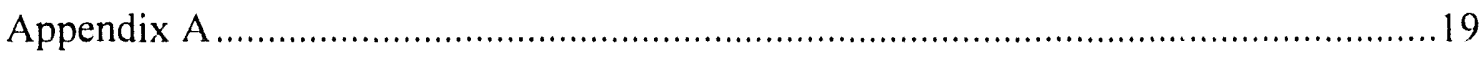

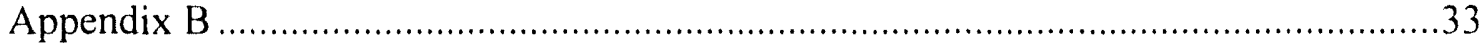

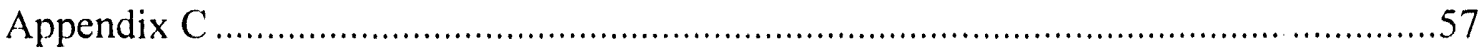

\section{FIGURES}

1. Crisp boundaries for measurement values ......................................................8

2 Fuzzy boundaries for measurement values ..................................................... 8

3. Decision curve used in program................................................................ 11

4. Flowchart of software used in PC simulation .................................................... 12

5 System level flowchart of the soft gre used in the hardware application ..................15

\section{TABLES}

1. An example of the relationship between major and minor data .............................

2. Bit usage of each 16 bit data word transmitted ............................................ 10

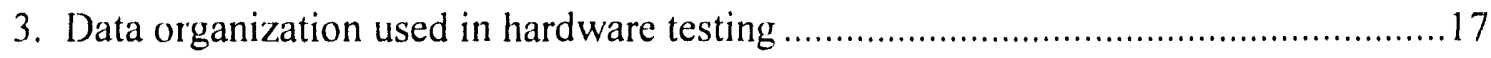




\section{REENTRY VEHICLE ADAPTIVE TELEMETRY}

\section{Introduction}

In RF telemetry the allowable RF bandwidth limits the amount of data in the telemetered data set. Typically the data set is configured to focus on the expected events at the expense of the unexpected events, such as system malfunctions. This is particularly true of diagnostic data since the customer is only interested in their data, not system diagnostics.

System diagnostics are further compromised because full diagnostics require large amounts of data and the data is only interesting in the event of a malfunction. Therefore diagnostics are typically reduced to a bare minimum. Unfortunately if a malfunction does occur, diagnosis of the problem is complicated by the fact that only a small subset of the telemetered diagnostics actually addresses any given malfunction. Consequently there is never sufficient diagnostic data when problems occur.

Diagnostics are not the only data that is compromised due to RF bandwidth limits. Since it is not uncommon for data requirements to change during phases of the test, a fixed data set is a compromise among the data needed in all phases. In the past either a less-than-optimal data set was used to cover all phases of the test, or several TM packages were used to cover different phases of the test.

One solution to these compromises is to design intelligence into the TM so it can adapt to changing data needs. During various phases of a test, the data set could change to reflect the current needs. If a malfunction is suspected, the TM could focus the diagnostic data to better cover the problem area. By using an adaptive TM, critical data transmission is ensured and the data set is optimized, resulting in reduced data rate and bandwidth. This reduction in bandwidth increases the system's operating range and make it easier to use with encryption techniques.

In this report, the problem of insufficient diagnostic data is addressed. Fuzzy Logic is used to drive an inference engine that determines which diagnostic data should be transmitted and at what data rate, while keeping the bandwidth dedicated to diagnostics constant. The first section (THEORY) briefly covers the theory behind the algorithm. The second section (IMPLEMENTATION) outlines how the algorithm was implemented. The third section (SOFTWARE SIMULATION) covers the software simulation performed on a PC and points out the differences between the simulation software and the software used to implement the algorithm in the final TM hardware package. The fourth section (HARDWARE IMPLEMENTATION) describes the hardware and software used to test the adaptive TM on a TM hardware system. The fifth section (TEST RESULTS) describes the results of the hardware testing. The sixth section (CONCLUSIONS) summarizes the results and implications of the tests. 


\section{Theory}

Figure 1 shows the a typical representation of boundaries between sets. In this example, a measurement is considered normal, too high, or too low. These boundaries are crisp, meaning a measurement is completely in one set and not at all in the other two sets. The transition from one set to another is abrupt. The only way to make these transition seem smoother is to break the sets into more subsets. However, the burden of computing a decision rapidly grows as the number of sets are increased.

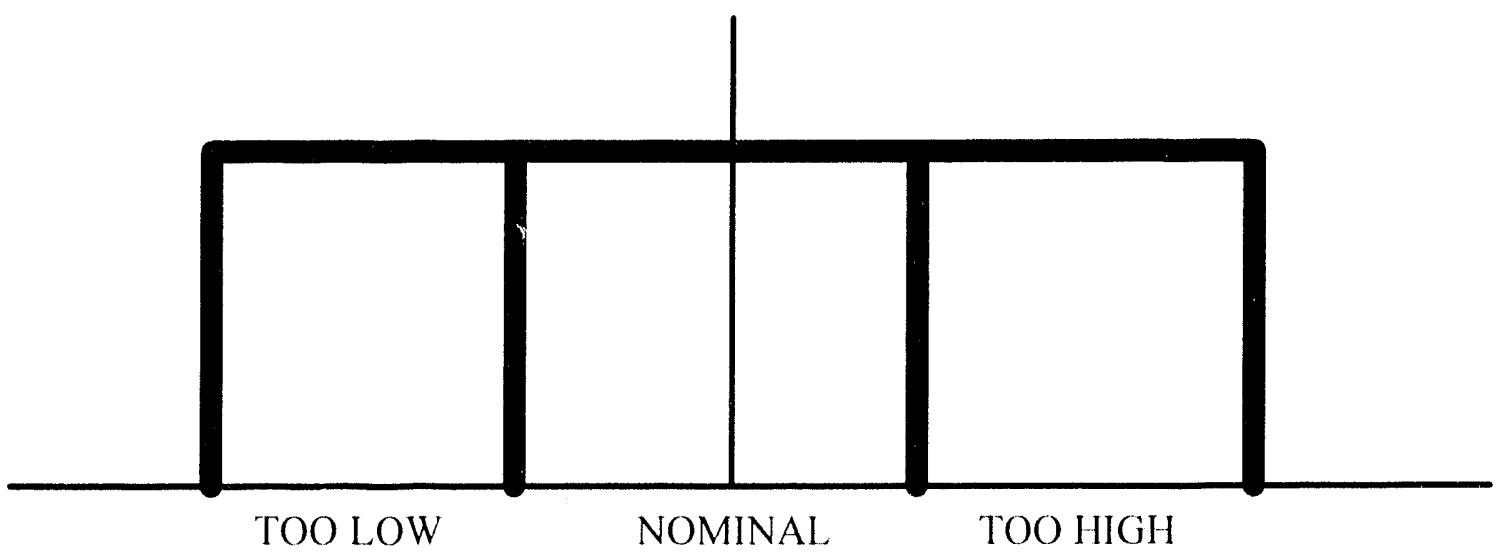

Figure 1. Crisp boundaries for measurement values.

Fortunately, in the adaptive telemetry application, precise sets of values are not required. Exploiting this tolerance for imprecision allows the sets boundaries to be approximated, or made fuzzy. In Figure 2, the three sets shown in Figure 1 are drawn with new boundaries. These boundaries are not abrupt and overlap.

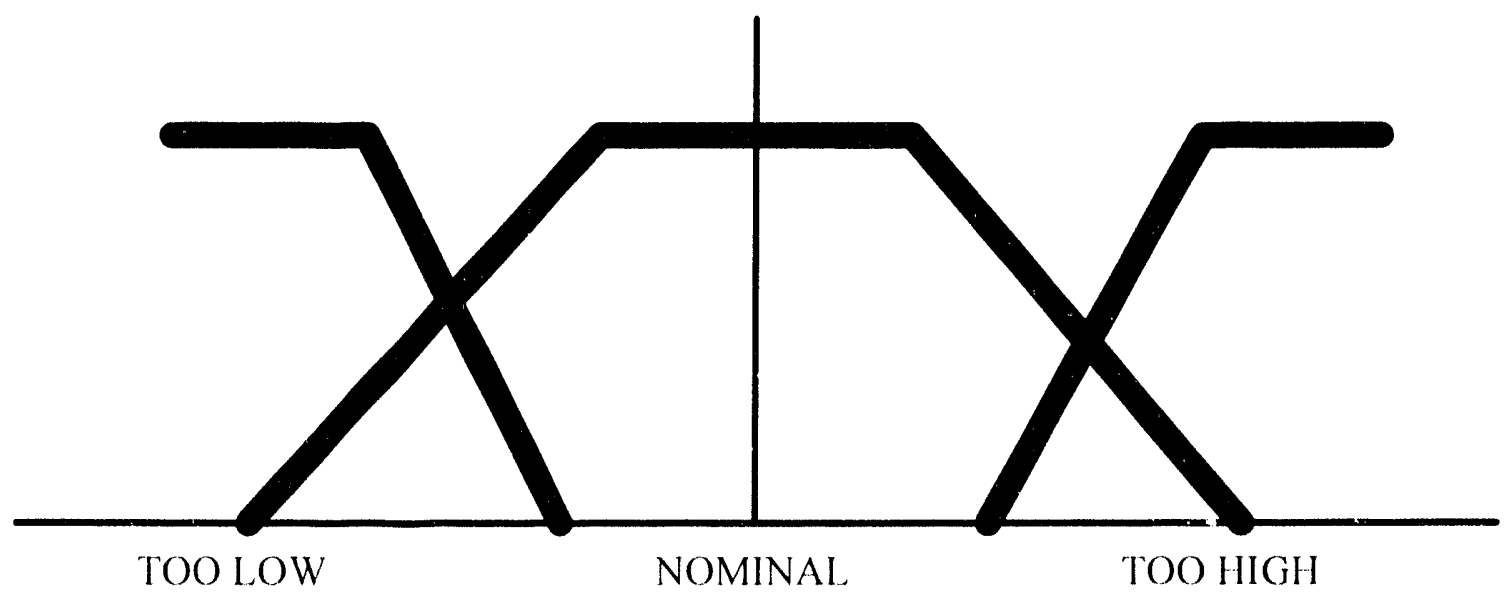

Figure 2. Fuzzy boundaries for measurement values. 
The reasoning behind overlapping the set boundaries is to allow for a smoother transition from one set to another without increasing the number of sets. A slight increase in the number of sets dramatically increases the required computational power. The logic required to implement these overlapping sets is referred to as fuzzy logic. Using fuzzy logic to build a fuzzy inference engine allows more intelligence to be programmed in a particular hardware configuration since the number of sets remain low.

The data used by the adaptive telemetry is divided into two categories: major and minor data. Each major data has zero or more minor data within its data group. The major data is always transmitted, and the minor data is only transmitted if the major data is outside of its nominal specifications. Using this scheme, a representative sample (the major data) from each process being monitored is telemetered. When a major measurement is determined to be outside of normal parameters, its entire data group is telemetered, thus increasing the amount of telemetered data concerning the problem area. An example of this arrangement is shown in Table 1.

\begin{tabular}{|c|c|c|}
\hline Temp 1 & Volts 1 & Temp 2 \\
\hline Voltage $\mathrm{a}$ & Current & \\
\hline Voltage $\mathrm{b}$ & Temperature & \\
\hline Voltage $\mathrm{c}$ & & \\
\hline Current $\mathrm{a}$ & & \\
\hline Current $\mathrm{b}$ & & \\
\hline
\end{tabular}

Table 1. An example of the relationship between major and minor data. Here there are three major data measurements: Temp 1, Volt 1, and Temp 2. In this example major data Temp 1 is part of a data group that has five minor data: Voltage a. Voltage b, Voltage c. Current a, and Current b. Major data group Volt 1 has two minor data, and major data group Temp 2 consists of only one major measurement and no minor data.

The fuzzy inference engine makes several decisions based on the actual measurements of the major data. First it determines if the measurements are within normal parameters. If they are, all major data measurements are transmitted with equal sample rates. When a measurement is determined to be outside of its normal parameters, the frame size is adjusted to accommodate its minor data group. As the data moves further outside its normal parameters, the sample rate of the data group is iricreased relative to the other groups. Again the frame size is adjusted to accommodate the increased sampling rate.

The output of the inference engine is used to construct a list of measurement values to be transmitted. Each word in the list is 16 bits. Since the order and length of this list may change, it is necessary to tag each measurement with a code identifying each measurement. 
Each 16 bit word consists of a tag code in the 6 most significant bits, and measurement data in the 10 least significant bits (Table 2). Using 6 bits for identification limits the number of unique measurements, both major and minor, in a list to 64 . However, the total number of lists is not limited except, perhaps, by implementation restrictions. Limiting factors include the computational speed of the inference engine, and the number of slots allotted for in each major frame. The length of the list is determined by the number of data and the sample rate of the data.

\begin{tabular}{||c|c|c|c|c|c|c|c|c|c|c|c|c|c|c|c|}
\hline 15 & 14 & 13 & 12 & 11 & 10 & 9 & 8 & 7 & 6 & 5 & 4 & 3 & 2 & 1 & 0 \\
\hline \multicolumn{3}{|c|}{ TAG CODE } & \multicolumn{8}{|c|}{10 BIT MEASUREMENT DATA } \\
\hline
\end{tabular}

Table 2. Bit usage of each 16 bit data word transmitted.

\section{Implementation}

To take further advantage of the tolerance of imprecision, several restrictions are placed on the fuzzy inference engine. First, only 16 bit integer math is used. All values are scaled to take full advantage of the precision allowed for in 16 bits. Secondly, the inference engine was designed to run on the absolute value of the difference between the nominal value and the actual value. This allowed the fuzzy data set to be reduced to half at the expense of requiring that the remaining data set be symmetrical about some center value. This center value is considered the nominal value. Finally, the inference engine only accounts for the off nominal deviations. If the actual value is within a certain amount of the nominal value, no calculations are made. This amount of variance around the nominal value is referred to (in this report) as the minimum range. Figure 3 shows the simplified fuzzy data set implemented in this program. Here the nominal value is the vertical axis. The minimum range is the range from the vertical axis to the point "A." The point " $B$ " is referred to as the maximum range. All values are predetermined to fall within the range of nominal to the point "B." As shown in Figure 3, all values beyond point "B" are clipped. Values beyond point "B" are still valid as far as the algorithm is concerned, but the values are treated the same as the value at "B." 


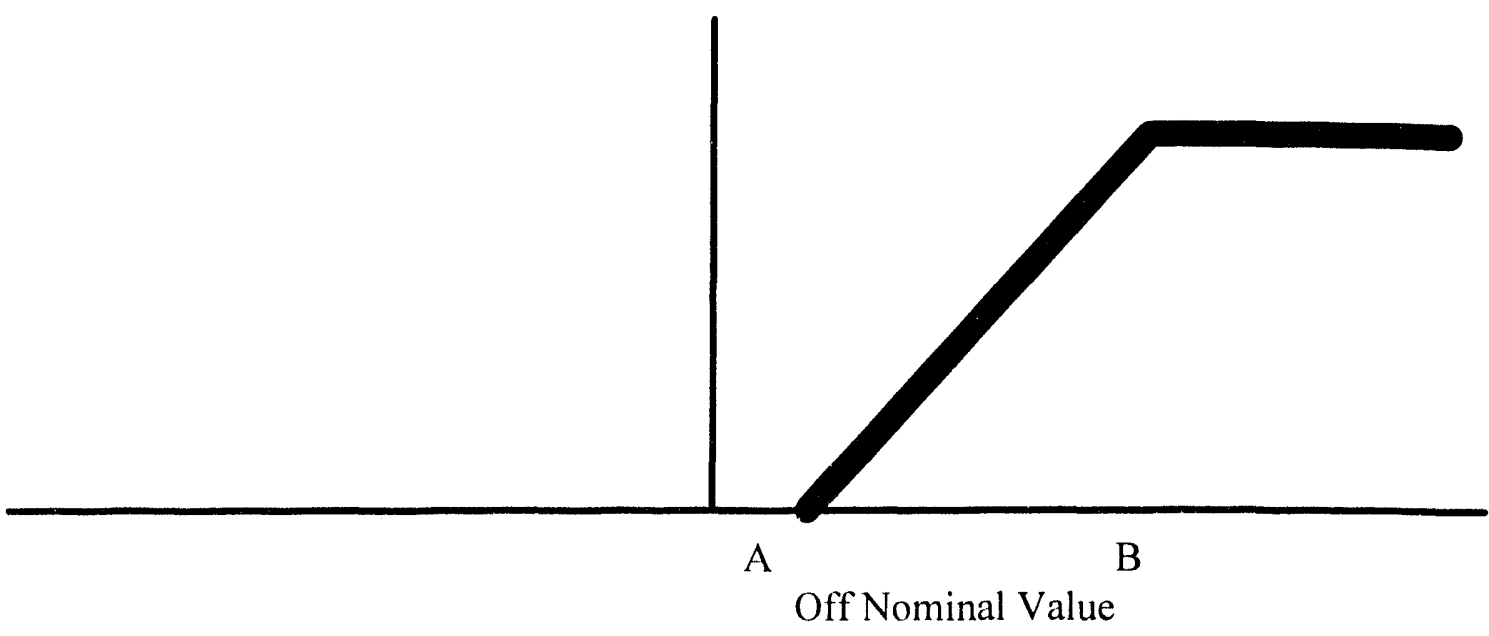

Figure 3. Decision curve used in program.

In order to implement the idea of a data list, the single data list is duplicated into two lists in this implementation. Each list alternates between the active list and the inactive list. The active list contains the list of measurements currently being transmitted. Each time the routine timer tick is called through a hardware interrupt, one measurement is updated and sent to the transmitter. When the last measurement in the list is read, the currently active list becomes inactive, and the currently inactive list becomes the active list. Once the lists change, the software invokes the fuzzy inference engine. This set of routines work in the background, computing a new list based on the data in the inactive list. The newly computed list is then written over the old, inactive list. The fuzzy inference engine then waits for the lists to change their active state again.

This dual list implementation results in a time lag in acting on measurements. When a measurement in the active list, say list \#1, is read, it is not acted upon until the end of the active list (list \#1) is reached. Then the data is used to generate a new list \#1 while the other list (list \#2) is being read and transmitted. This new list \#1 remains inactive until the active list (list \#2) is completed. When list \#2 is completed, list \#1 becomes active and the new data format is finally transmitted. This time lag affects only the data format and not the actual data, since the most current data is immediately transmitted.

Figure 4 shows a flow chart of the software developed to implement the inference engine used by the adaptive telemetry package. A description of each routine shown in the flow chart follows the flow chart. 
Read information concerning particulars of the measurement data

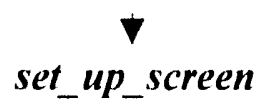

Set up computer screen to display simulation

\section{sloper}

Compute the slope of the fuzzy boundary from normal and off normal sets

\section{list extract}

Extract the latest data from the list

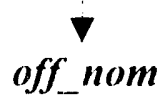

Using fuzzy logic, determine about how far off nominal each data is

$$
\text { rel freq }
$$

Compute how often each data measurement appears in the data list

$$
\stackrel{\nabla}{\text { stuff_list }}
$$

Create a list of measurement data

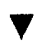

Wait here until the current acive list is finished being transmitted

Figure 4. Flowchart of software used in PC simulation.

\section{list_extract}

This routine is used both in the initialization and in the continuous loop. It removes the measurement data from the inactive list and places it into the measurement structure. This data is the most recent data gathered and is used to compute the next list of measurements to be transmitted.

\section{off nom}

This routine is used both in the initialization and in the continuous loop. It computes the fuzzy value associated with how far off nominal the actual value is. 


\section{read_data}

This routine is only used during system initialization. It reads from disk the data required to initialize the inference engine. This data includes the nominal value, the minimum and maximum expected deviations (points $\mathrm{A}$ and $\mathrm{B}$ in figure 3 ), and the size of the data group. It also reads in an initial value for each measurement. This value is inserted into both the active and the inactive list.

\section{rel freq}

This routine is used both in the initialization and in the continuous loop. It calculates how often each measurement will appear in the list. It computes this by comparing the fuzzy values computed in off_nom. This routine also computes the length of the list. The length of the list is limited to a maximum of 256 items. An upper limit on the length of the list was set to reduce the time between reading a value that will cause a change in the structure of the list, and transmitting a new list.

\section{set_up_screen}

This routine is only used during system initialization. It also is only used in the simulation software. Its function is to initialize the computer screen to display simulated data.

\section{sloper}

This routine is used only in initialization. It computes the slope of the line from the minimum to the maximum deviation.

\section{stuff_list}

This routine is used both in the initialization and in the continuous loop. It creates the list based on the relative frequencies computed in rel freq.

\section{timer tick}

This routine is only used in the continuous loop. It is an interrupt handler, and for simulation purposes, it is called by the PC-DOS timer tick. In an actual application, the interrupt would be set by the transmitting hardware when data is needed for transmission. This routine reads the latest available data for the measurement pointed to in the active list, and transmits down the data. (In the simulation, the data is displayed on the screen in the appropriate area.) This routine also senses the end of the active list, and makes the currently inactive list, active. This switch is detected by the software and the new inactive list is used to compute the next active list.

\section{Software simulation}

The program developed to simulate the adaptive telemetry system on a PC is named "adaptm.c." It mimics a hardware application as closely as possible. A listing of the program 
is given in Appendix A. This program and the PC it runs on proved to be a valuable platform for developing the adaptive TM algorithm. The program uses the PC-DOS $18 \mathrm{~ms}$ timer tick as an interrupt to trigger data "transmission." The data is then "decommutated" in software. Transmission and decommutation amounts to creating and then independently deciphering the 6 bit indentification code (see table 2) and printing the data on the screen in a predetermined place. The data itself is initialized from a disk file. In order that the data be varied during execution, the routine main contains code to accept data from the PC's keyboard in the background.

\section{Hardware implementation}

The program "adaptm.c" listed in Appendix A was modified to operate on the ASDACS (Advanced Stored Data Acquisition Control System) hardware package. The ASDACS package is a general purpose system used by the Telemetry Technology Development Department for instrumentation. The hardware configuration chosen to test the adaptive TM is as follows:

$\begin{array}{ll}\text { CPU } & \text { Intel 80C196KC } \\ \text { A/D converter } & 10 \text { bit, 13.3uS } \\ \text { PCM Data output } & \text { NRZ-L } \\ \text { Main Memory } & 2 \mathrm{MB}\end{array}$

The data inputs were simulated using an array of 30 potentiometers and 24 switches. The potentiometers gave a continuously variable output from 0 to $5 \mathrm{VDC}$, while the switches provided bilevel signals of 0 or $5 \mathrm{VDC}$. These 54 signals were fed into a multiplexer/bilevel board controlled by the ASDACS. The variable output of the multiplexer/bilevel board was fed into the 10 bit A/D converter and the bilevel outputs of were read directiy by the ASDACS.

Shown below in Figure 5 is a system level flowchart of the modified software. This shows the algorithmic flow, as well as the interrupt path taken when the transmission buffer is empty. Since the hardware PCM transmitter is double buffered, the hardware is transmitting one word while another is being loaded into the transmission buffer. 


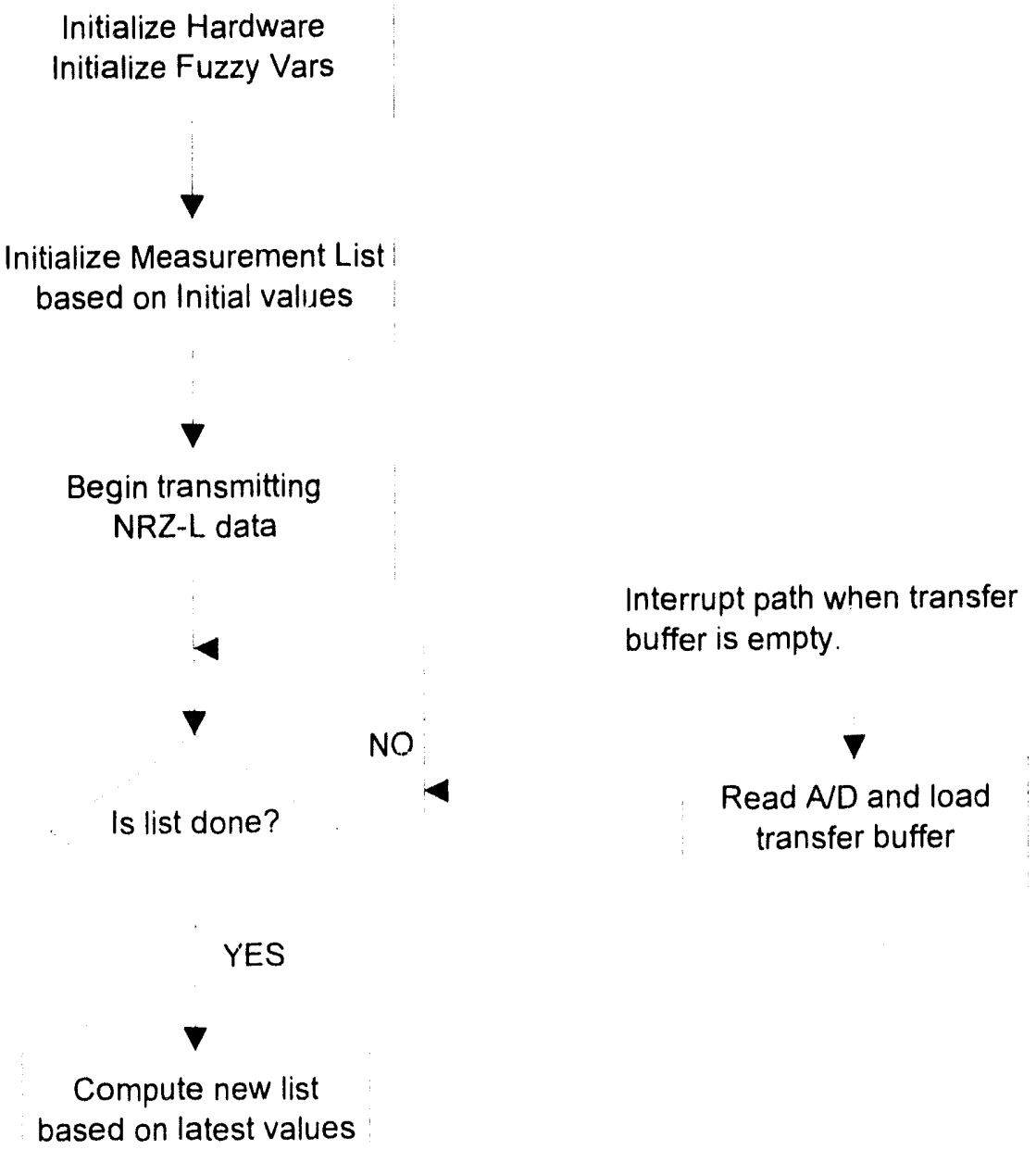

Figure 5 System level flowchart of the software used in the hardware application.

The modifications to "adaptm.c" listed in Appendix A are outlined below. The primary additions and modifications were made to accommodate the different platforms. The adaptive TM inference engine was not modified.

\section{read data}

This routine was eliminated since the initiaization data is stored in PROM and not on disk. The initialization of the variables was inserted in the variable initialization/definition section of the program. Here the data is inanualiy transferred from PROM to RAM on startup. 
set_up_screen

This routine was deleted since there is no video output.

timer_tick

This routine was replaced with the routine PCM_buffer_empty. This new routine is also interrupt driven. However it receives its interrupt signal from the PCM data transmission buffer when the buffer is empty. This routine then inserts the next data as determined by the data list.

\section{Init_Internal_A2D}

This routine was added to initialize the 10 bit $A / D$ hardware.

\section{Init CPU}

This routine was added to initialize the CPU hardware.

\section{Init_PCM}

This routine was added to initialize the PCM hardware.

\section{Init_Interrupt}

This routine was added to initialize the ASDACS hardware interrupts.

\section{Main}

This routine was modified to account for the new hardware and to remove the references to the PC keyboard entries. However, the algorithm for calling the adaptive TM routines were not changed.

\section{Test results}

To fully test the capabilities of the algorithm, 64 data inputs were needed. To accomplish this using the 54 outputs of the analog input array, 10 of the outputs were duplicated and used twice by the software. In software, 10 of the 64 possible measurements in the measurement list were remapped to 10 of the 54 inputs. All 20 of these duplicate signals (10 pairs) were bilevels and were used as minor data in various groups.

The 64 data inputs were grouped into 20 data groups. 18 of these groups had a variable input as their major data word, and 2 groups had a bilevel input as their major data word. The organization of the data groups is shown in Table 3. Also shown in Table 3 is the boundary conditions. A value is considered nominal if it fall within the limits of MIN. Once the value goes outside of the MIN limits, the inference engine considers the data to be off nominal and takes appropriate action. The action becomes more pronounced as the value approaches the limits of MAX. MAX is considered to be the farthest the value will ever be from nominal. 
For example, if group 2 in Table 3 were a set of sensors on a human body with the major measurement being internal body temperature, a temperature value within the bounds of MIN would not cause alarm. However if the body temperature exceeds 100 degrees, the patient requires closer attention. When this occurs the software adds the other measurements in the group to the list of data being transmitted. As the major measurement approaches 110 degrees, the group 2 data is transmitted more frequently. In this example, values beyond 110 degrees are never expected. However, if the value was to exceed 110 , the inference engine simply clips the value and treats the value as if it was 110 .

\begin{tabular}{|l|l|l|l|l|}
\hline GROUP\# & MIN & MAX & MAJOR & MINOR \\
\hline 1 & $500+-10$ & $500+-50$ & POT1 & POT19,SW3,SW4 \\
\hline 2 & $98+-2$ & $98+-12$ & POT2 & SW5,POT20,SW6,SW7,SW8 \\
\hline 3 & $100+-10$ & $100+-40$ & POT3 & \\
\hline 4 & $128+-32$ & $128+-110$ & POT4 & SW9,SW10 \\
\hline 5 & $110+-2$ & $110+-5$ & POT5 & POT21,SW11,POT22,SW12 \\
\hline 6 & $900+-100$ & $900+-300$ & POT6 & SW13 \\
\hline 7 & $75+-45$ & $75+-60$ & POT7 & \\
\hline 8 & $10+-5$ & $10+-10$ & POT8 & \\
\hline 9 & $512+-500$ & $512+-999$ & POT9 & SW14,POT23,SW15 \\
\hline 10 & $180+-30$ & $180+-35$ & POT10 & POT25,SW16,SW17,SW18,SW19 \\
\hline 11 & $645+-80$ & $645+-140$ & POT11 & SW20 \\
\hline 12 & $120+-40$ & $120+-80$ & POT12 & SW21,SW22,SW23 \\
\hline 13 & $100+-5$ & $100+-100$ & POT13 & SW24,SW3,SW4 \\
\hline 14 & $80+-20$ & $80+-50$ & POT14 & \\
\hline 15 & $780+-130$ & $780+-160$ & POT15 & SW5,SW6 \\
\hline 16 & $30+-20$ & $30+-40$ & POT16 & POT26,SW7,SW8,SW9 \\
\hline 17 & $30+-30$ & $30+-60$ & POT17 & SW10 \\
\hline 18 & $230+-30$ & $230+-60$ & POT18 & SW11,SW12,POT27,SW13,POT28 \\
\hline 19 & $1+-10$ & $1+-15$ & SW1 & POT29,POT30 \\
\hline 20 & $1022+-100$ & $1022+-120$ & SW2 & \\
\hline
\end{tabular}

Table 3. Data organization used in hardware testing.

Testing was performed at a relatively slow data rate to enable the observer to watch the reactions of the system. The system was tested by varying one or more of the analog inputs and observing the change in data and in data rate. Since the TM output was decommutated in real time, the effects could be seen on the video screen of the decommutating PC. Using this set up, we were able to exercise the algorithms ability to adapt to changing data requirements. The inputs were randomly varied in and out of the normal range while observing the system's reaction. Even a worse case scenario was performed with all data off normal to test the algorithm's conflict resolution capability. No problems were noted. Also the system was tested with all but one group off normal to ensure that the one normal group would still be sampled, if only occasionally. 
Although the system was primarily tested at a data rate of $1 \mathrm{~kb} / \mathrm{s}$, the system as configured operated successfully at data rates up to $100 \mathrm{~kb} / \mathrm{s}$. At speeds greater than $100 \mathrm{~kb} / \mathrm{s}$ the ASDACS could no longer compute a new list before the old list was transmitted. Even though this speed far exceeds the typical diagnostic data channel, much higher speeds could be achieved by some simple changes. First of all, no attempts were made to stream line the software. It was written as a demonstration of an idea. Secondly the overhead associated with the demonstration software is unrealistic. A real TM system would have more than one data channel per frame. This system only has one data channel. This means all the overhead is dumped on one data channel rather than being spread out among many more channels. Even if this system were being used as one channel in a larger system, the 32 bits of the sync pattern and the SFID would not be present.

\section{Conclusion}

The testing demonstrated the ability of the algorithm to detect an out-of-normal condition and make an intelligent decision on what data is the most important. Due to the fuzzy logic the algorithm transitioned smoothly from one condition to the next. The algorithm had no problems handling more than one out-of-normal condition. Again, fuzzy logic assisted in the algorithm's conflict resolution capability.

If this system were used in an actual flight, its ability to focus the diagnostic data on the problem(s) at hand would greatly increase the value of the transmitted diagnostic data without increasing the required bandwidth.

Although this test focused on diagnostic data, the data could just as easily have been other flight data. This ability to fine tune the transmitted data could prove useful in focusing flight information on specific parts of the flight. This would increase the total amount of different flight data without increasing the required bandwidth. 


\section{Appendix A}

The following is a program listing of the software used in the PC simulation of the adaptive TM. 


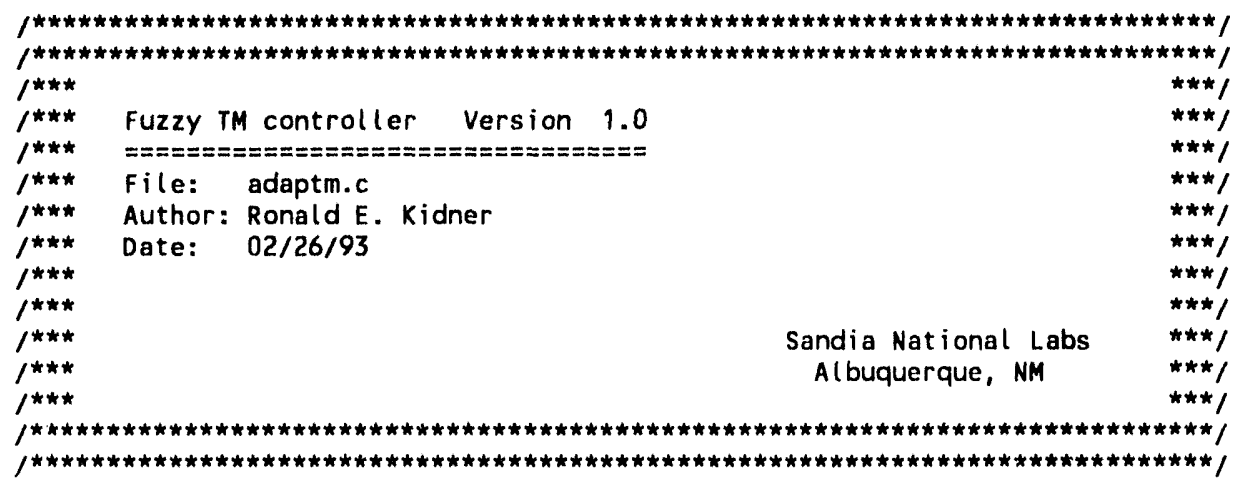

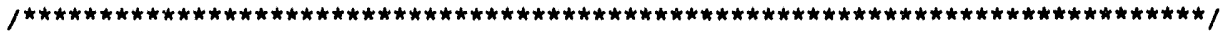

l* Include Files $\quad *$ I

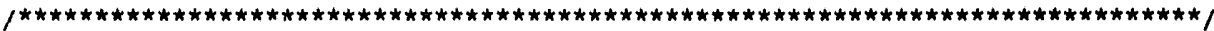

\#include 〈graph.h>

\#include <stdio.h>

\#include <math.h>

\#include $\langle i o . h\rangle$

\#include <stdlib.h>

$\#$ include <dos.h>

$\#$ include <conio.h>

\#include <string.h>

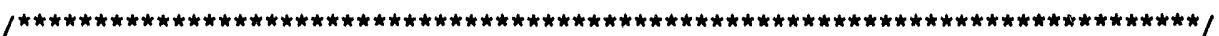

$I^{*}$ Define Definitions

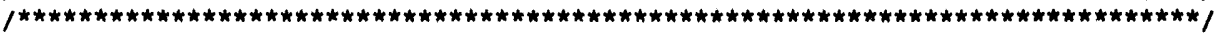

\#define max_priority_range $30 /$ * user defined max difference from lowest *

$l^{*}$ computed priority to highest priority *I

\#define max_priority_divisor 30000/max_priority_range

\#define max_list_len $256 /{ }^{*} \max$ possible len of list */

\#define num_of_groups $\quad 2 /^{*}$ number of groups of data. Each group */

1 * occupies one location in major frame */

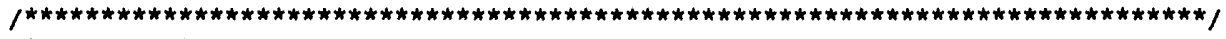

$I^{*}$ External Functions */

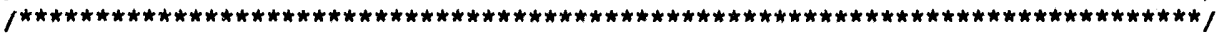

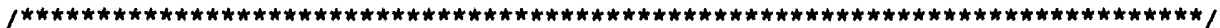

$f^{*}$ Internal Functions $\quad$ /

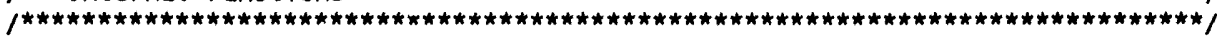

void list_extract(void);

void off nom(void);

void read_data(void);

void rel_freq(void);

void set up screen(void);

void sloper(void);

void stuff list(void);

void inter rupt far timer_tick(void);

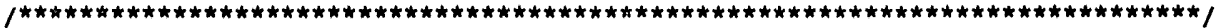

l* Internal Variables

char buff[80], buff1[80], buff2[5], buff3[5];

void far *old_timer;

int last_loc,present_loc, tick_count;

int $t i, t \bar{j}, t k, t l$;

int far *screen;

int screen_off: 


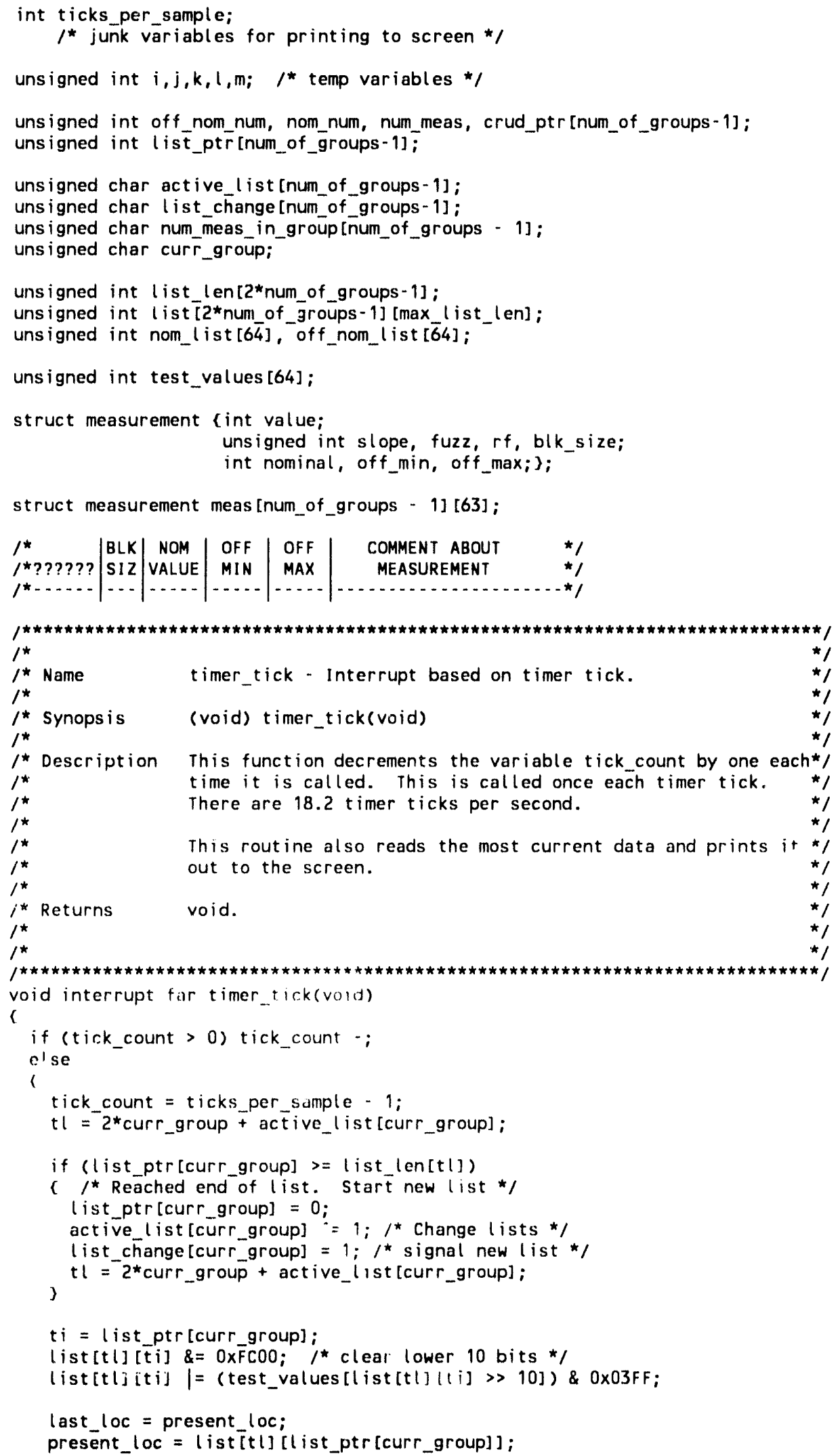




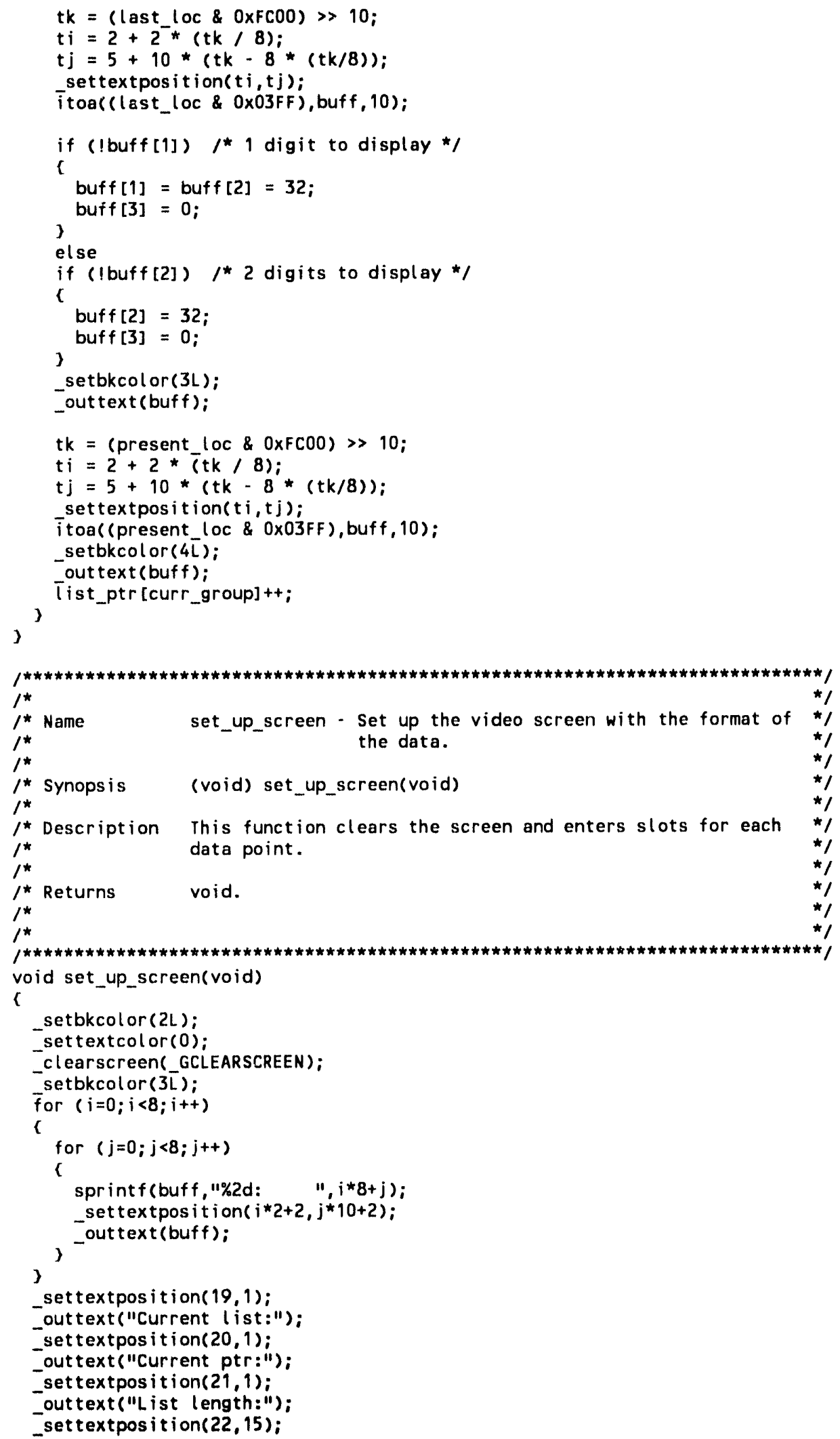




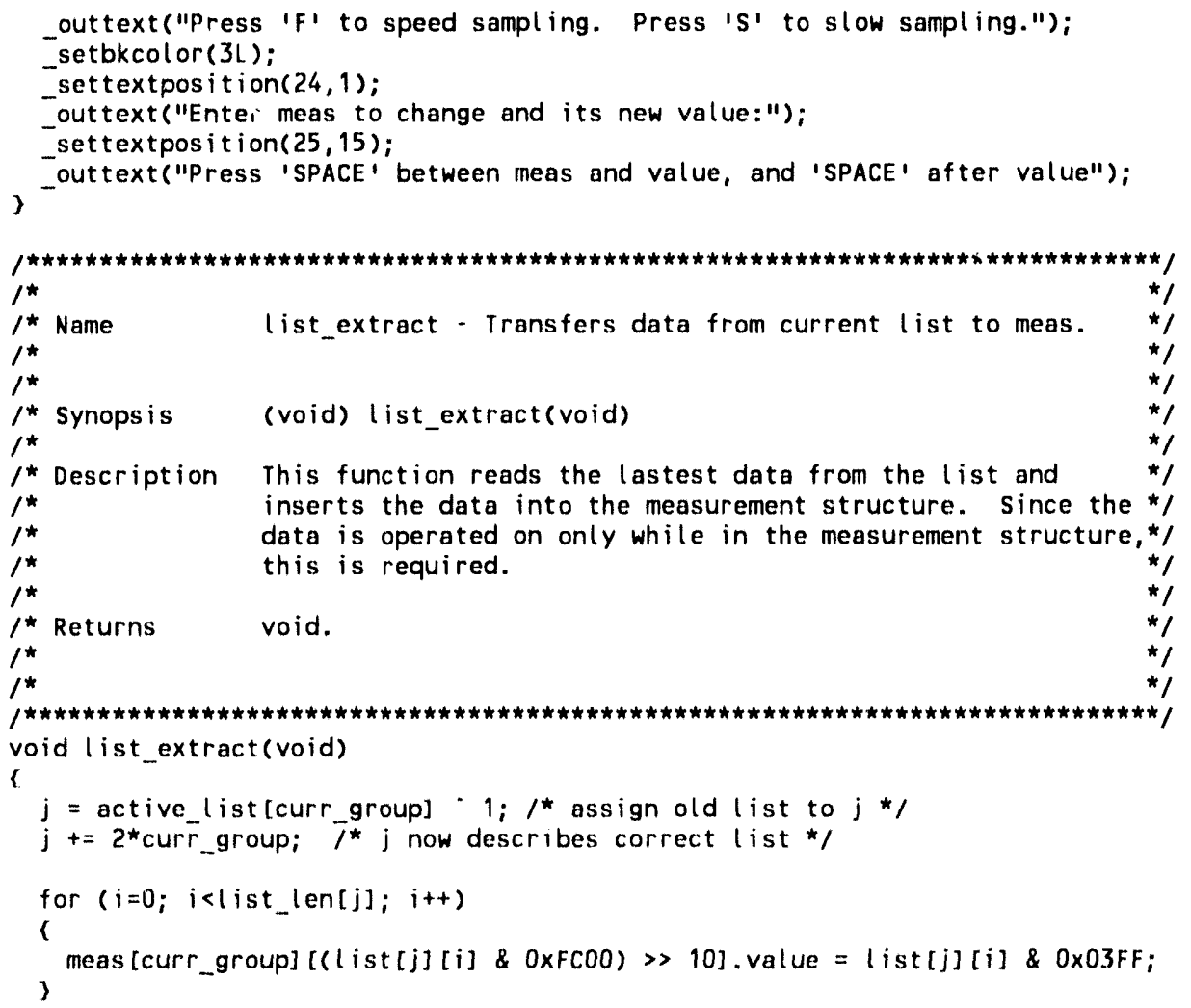



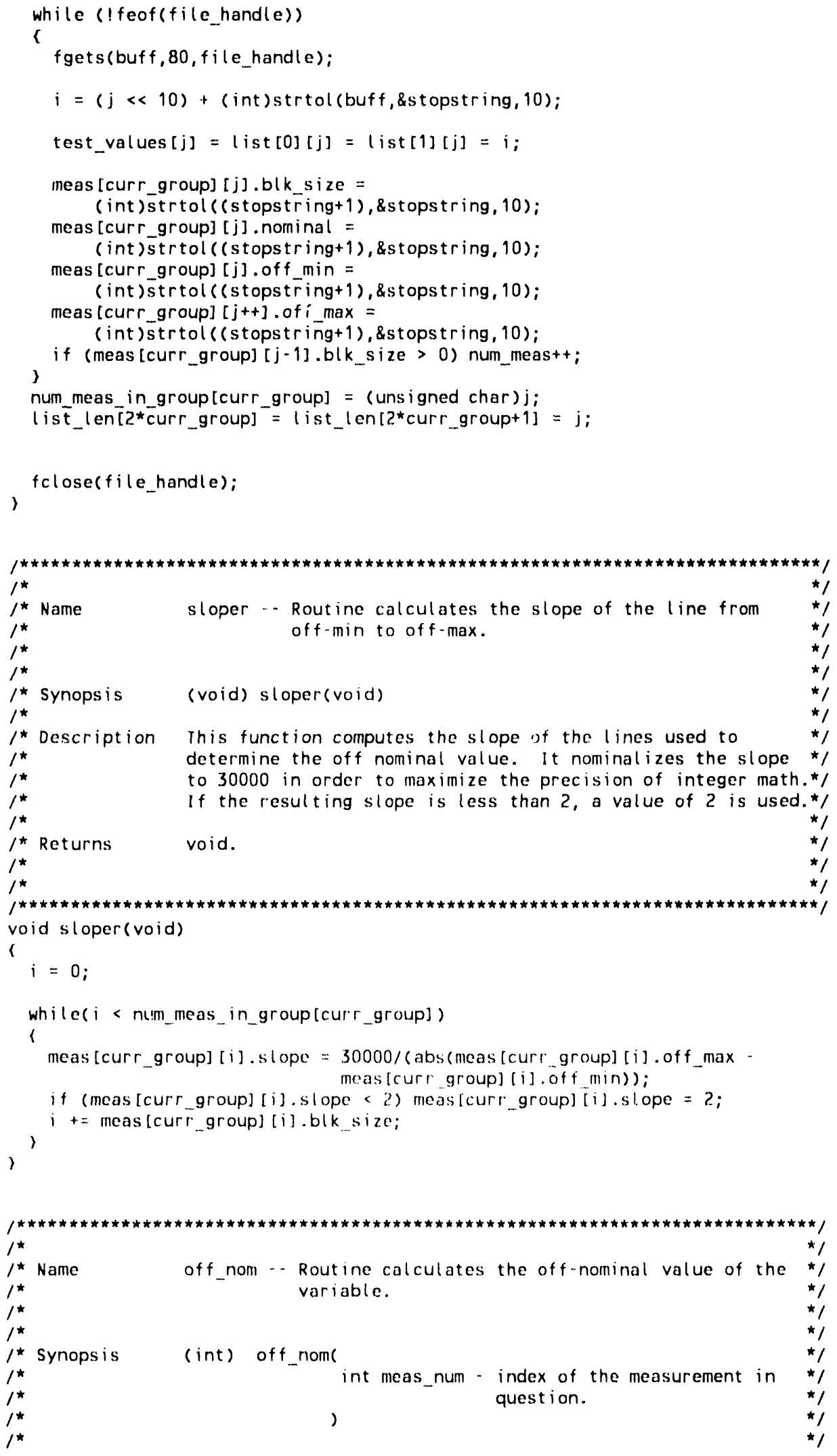


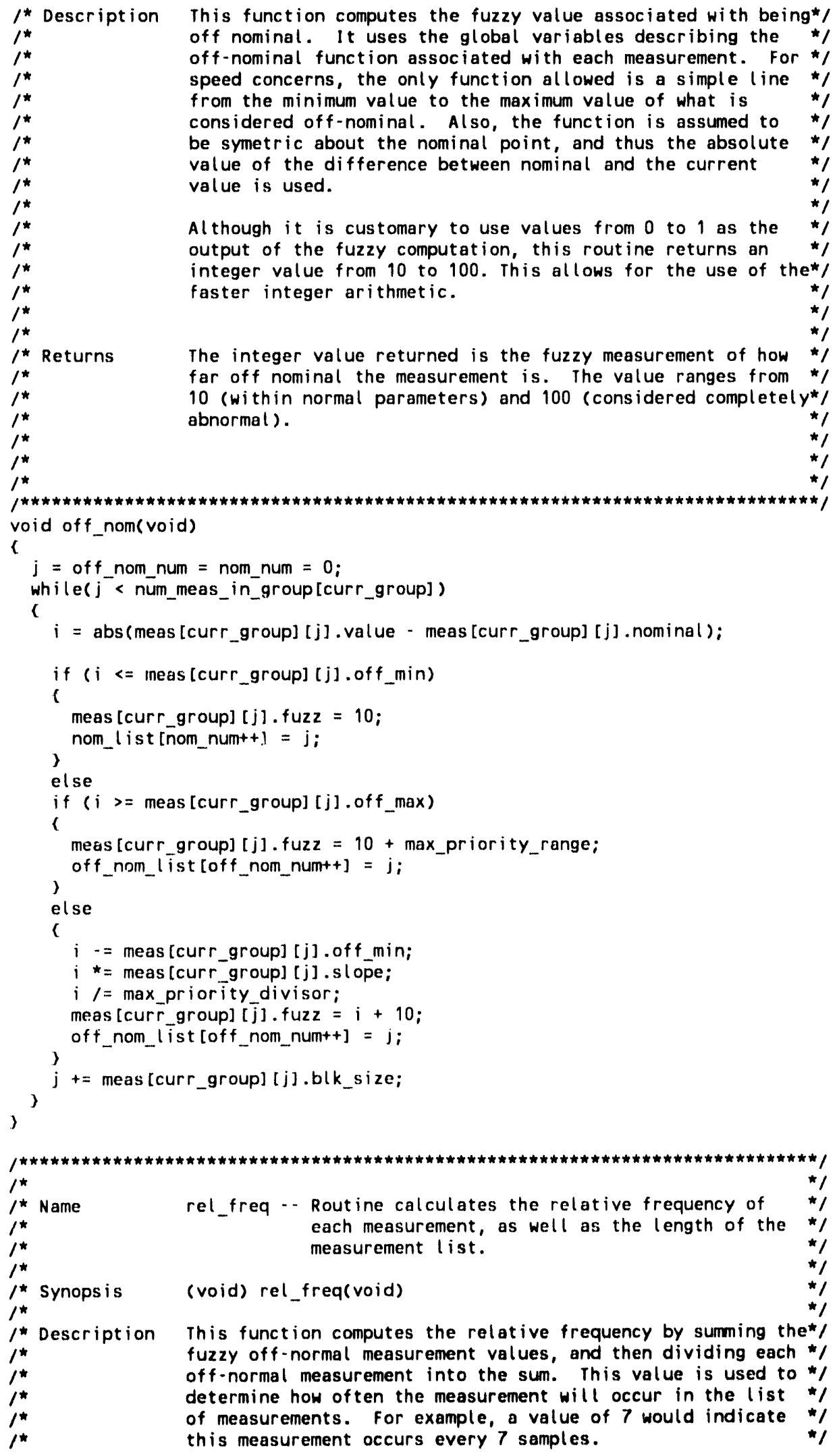




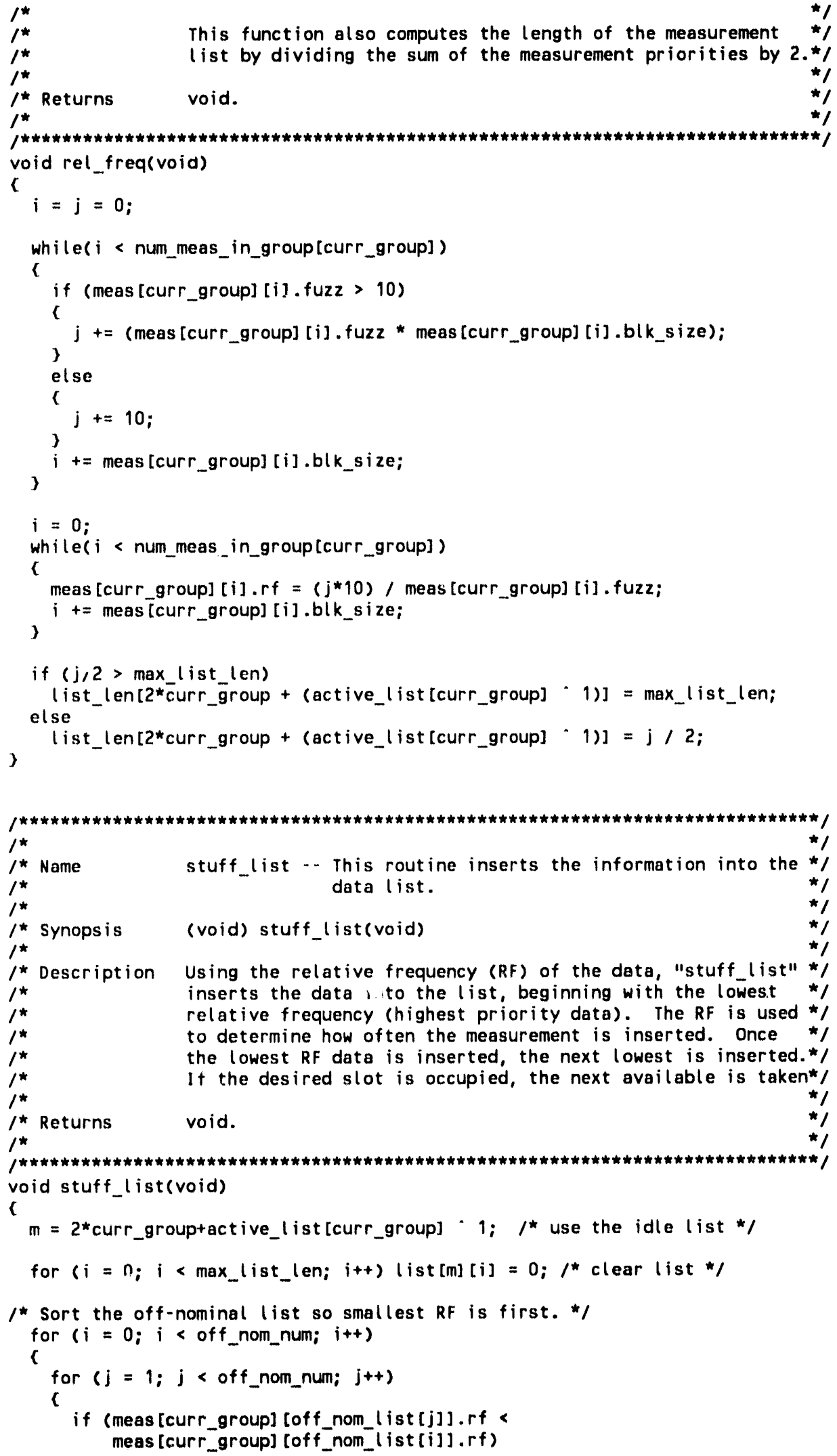




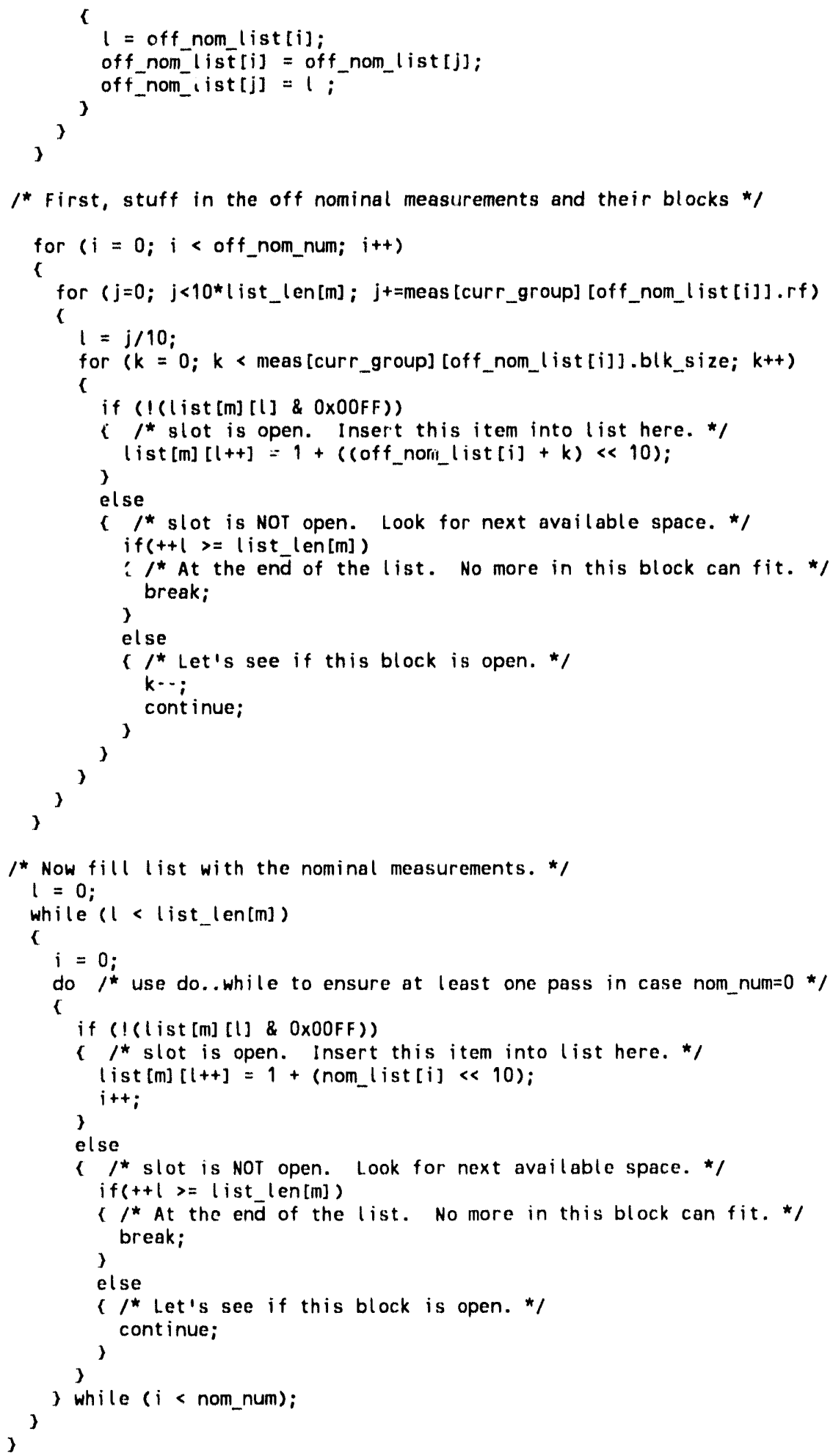




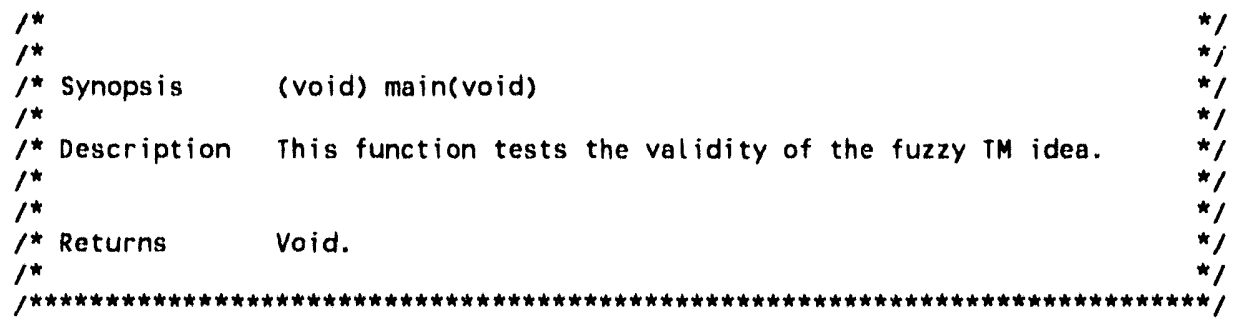

void main(void)

s

int va! int, sig_int, sig_val, the ptr, char_position;

char val_char [10], sig_char [10], inp_char;

the_pt $r=$ sig_val $=$ present_loc $=$ curr_group $=0$;

val_int $=1$;

char._position $=42$;

screen $=$ (int far ${ }^{\star}$ ) $0 \times 688000000$

screen_off $=0$;

for $(i=0 ; i<$ num_of_groups; $i++)$

亿

active $l$ ist $[i]=0$

list_ptr[i] $=0_{i}$

list_change $[i]=0$;

val char [0] $=0$;

sig_char $[0]=0$;

ticks_per_sample $=1 ; I^{*}$ default number of ticks per sample *

read_data(); $l^{*}$ for testing, read initial data from disk */

set_up_screen();

old_timer $=$ dos_getvect $(0 \times 10) ; / *$ save current timer tick */

sloper():

list extract();

off_nom();

rel freq();

stuff_list();

_dos_setvect $(0 \times 10$, timer_tick);

while (val_int)

if ( 1 ist_change [curr_group])

list_extract();

off nom( ):

rel_freq();

stuff_list( );

list_change [curr_group] $=0$;

itoa(active_list [curr_group], buf $f 1,10$ );

outp(0x21, 1)

_settextposition $(19,15)$;

out text (buff1);

outp $(0 \times 21,0)$;

itoa(list_len [2*curr_group + active_list [curr_group] ], buff 1,10$)$;

if (I buff $\overline{1}[2]$ ) 


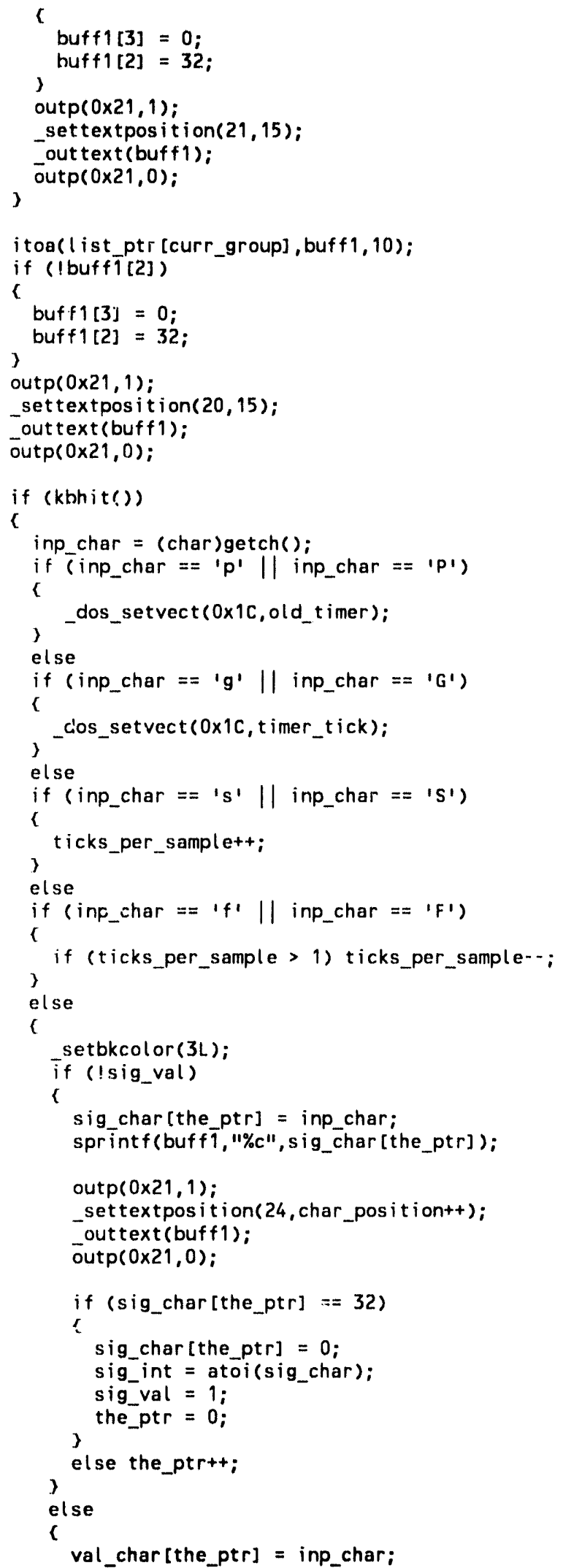




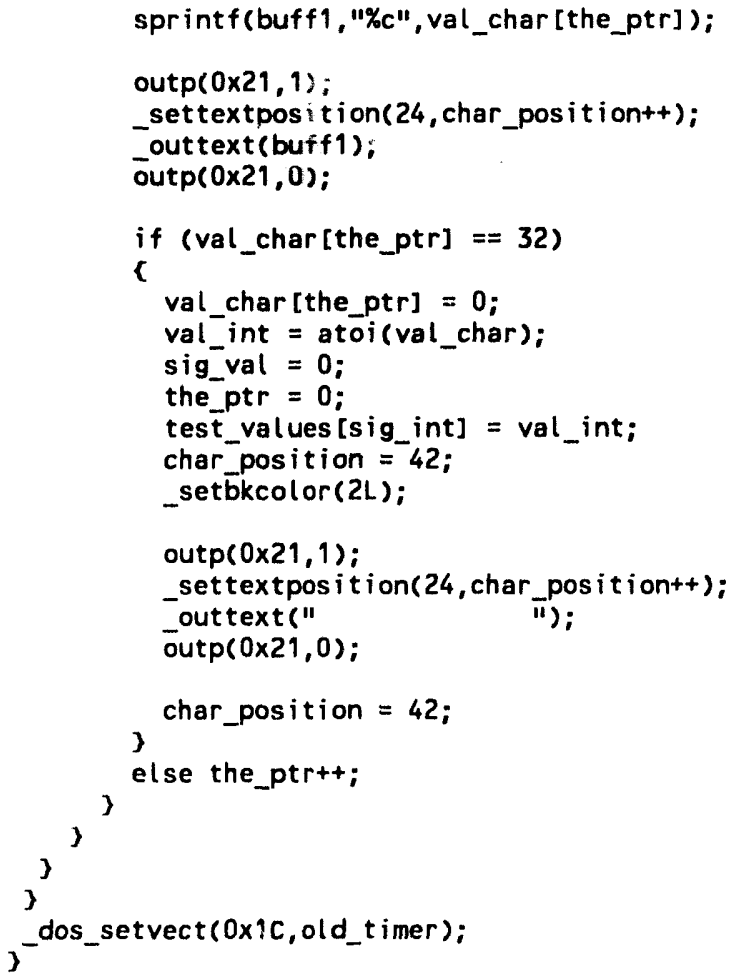




\section{Appendix B}

The following is a program listing of software used to drive the ASDACS harware package. 


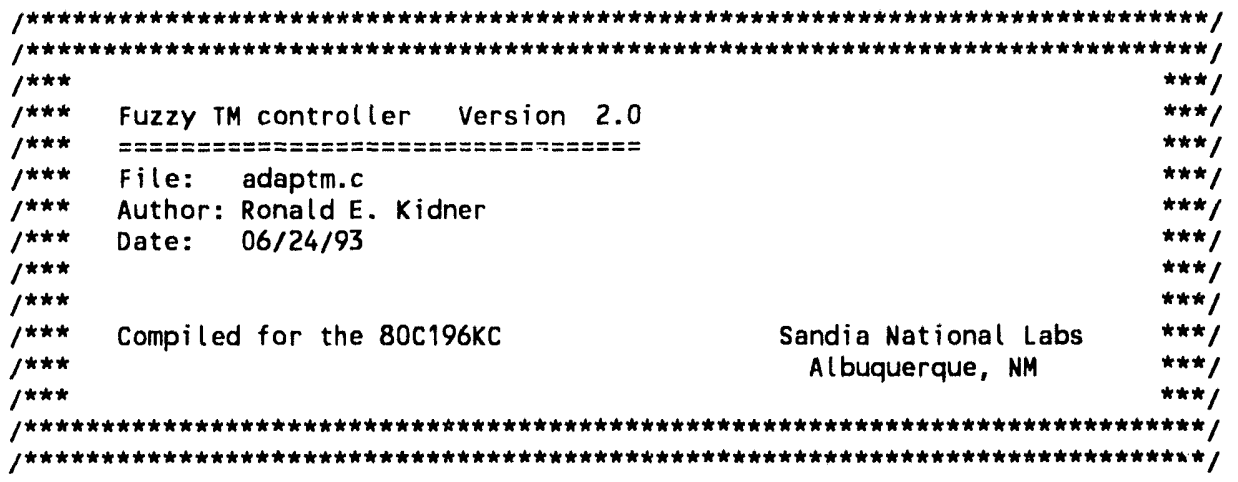

\#pragma ccb(OxEF) $/ *$ set the chip configuration register */

\#pragma interrupt (PCM_buffer_empty $=7$ ) $/$ * External Interrupt */

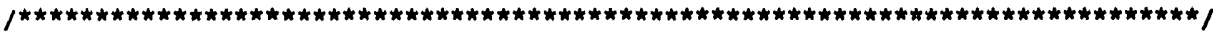

/* Include Files

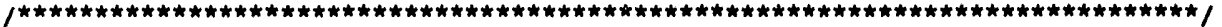

\#include $<80 \mathrm{c} 196 . \mathrm{h}\rangle$

\#include "sd addr.h"

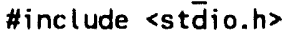

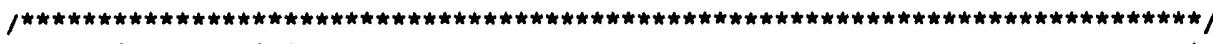

$1 *$ Define Definitions

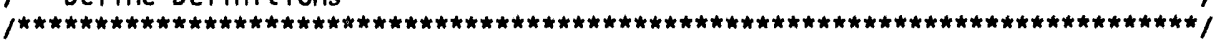

\#define max_priority_range $30 /$ user defined max difference from lowest *

/* computed priority to highest priority */

\#define max_priority_divisor 30000/max_priority_range

\#define max_list_len $256 / /^{*} \max$ possible len of list */

\#define num_meas_in_group $64 /$ * total meas in the group */

\#define num_of_groups

$2 I^{*}$ number of groups of data. Each group */

$l^{*}$ occupies one location in major frame */

$1 *$ Each group requires two slots, so that */

$1 *$ for 1 group, enter 2 here. For 3 groups*/

$1^{\star}$ enter 6 here.

$\begin{array}{ll}\text { \#define value } & 0 \\ \text { \#define slope } & 1 \\ \text { \#define fuzz } & 2 \\ \text { \#define rf } & 3 \\ \text { \#define blk_size } & 4 \\ \text { \#define nominal } & 5 \\ \text { \#define off_min } & 6 \\ \text { \#define off_max } & 7\end{array}$

unsigned int BILEVEL1; $/$ * Location of bilevel buffer \#1 (16 bit) */

\#pragma locate (BILEVELI = OXFFOC)

unsigned int BILEVEL2; /* Location of bilevel buffer \#2 (8 bit) */

\#pragma locate (BILEVEL2 = OXFFOA)

unsigned int MUXSET; 1 * Location of mux control channel */

\#pragma locate (MUXSET $=$ OXFFO8)

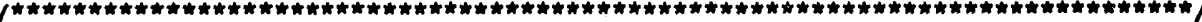

$I^{*}$ External Functions $\quad$ /

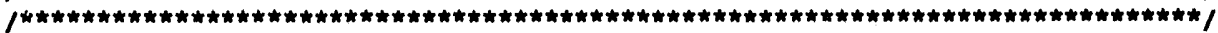

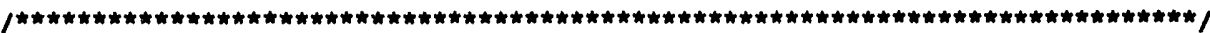




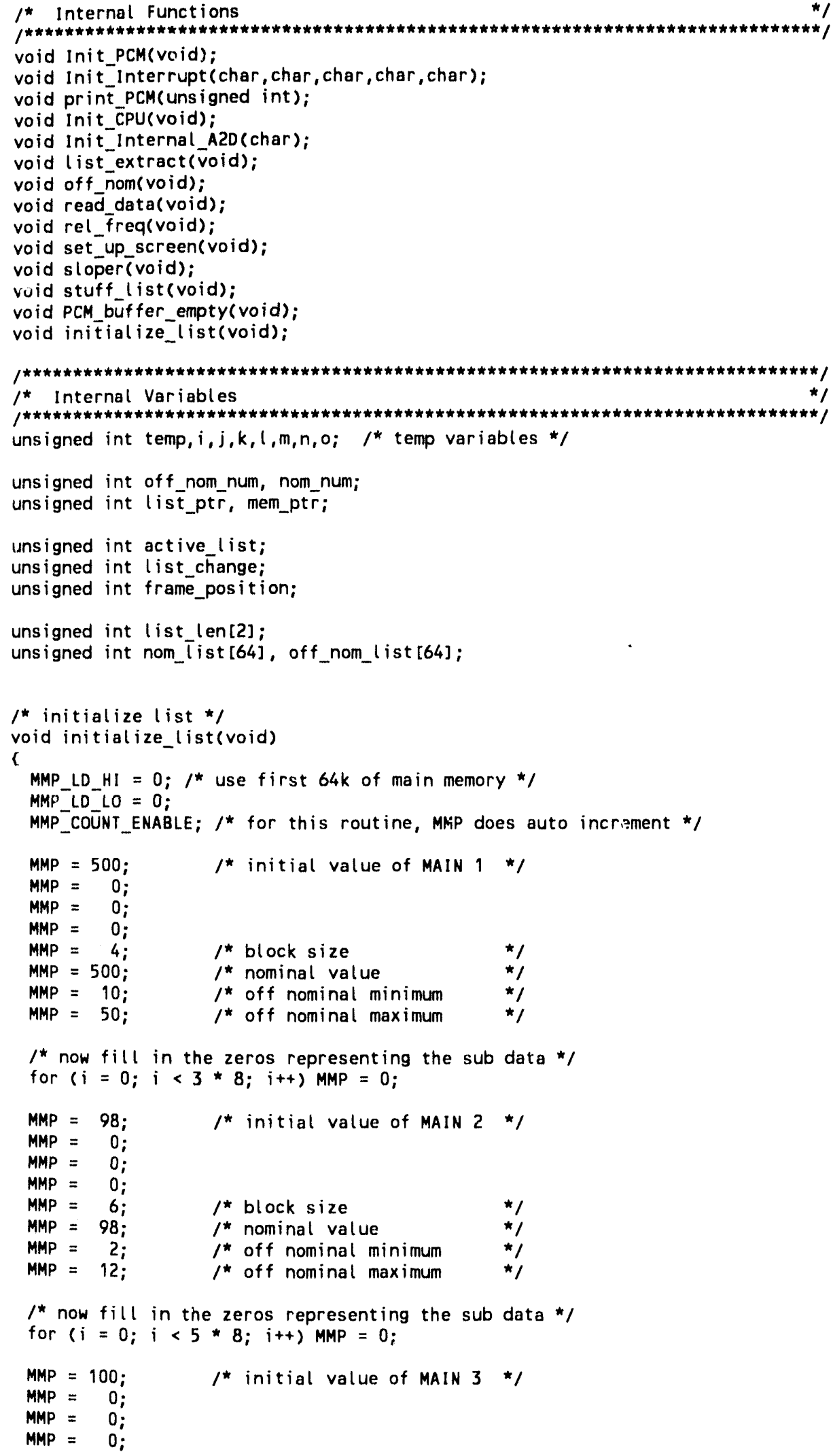

$I^{*}$ now fill in the zeros representing the sub data * for $(i=0 ; i<3 * 8 ; i++) M M P=0$;

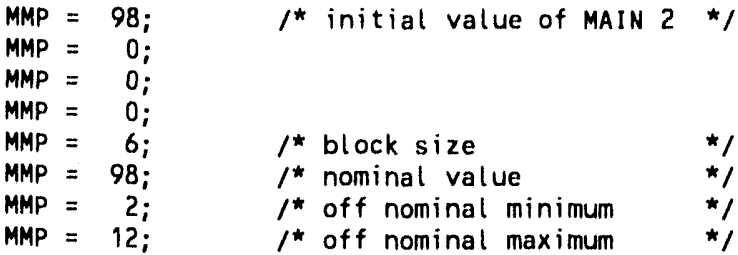

$I^{\star}$ now fill in the zeros representing the sub data $/$ for $(i=0 ; i<5 \star 8 ; i++)$ MMP $=0$;

MMP $=100 ; \quad l^{*}$ initial value of MAIN $3 * /$

MMP $=0$

MMP $=0 ;$

MMP $=0 ;$ 


$\begin{array}{ll}\text { MMP }=1 ; & / \star \text { block size } \\ \text { MMP }=100 ; & / * \text { nominal value } \\ \text { MMP }=10 ; & / * \text { off nominal minimum } \\ \text { MMP }=40 ; & / * \text { off nominal maximum }\end{array}$

$l^{*}$ now fill in the zeros representing the sub data */ for $(i=0 ; i<0 * 8 ; i++)$ MMP $=0$;

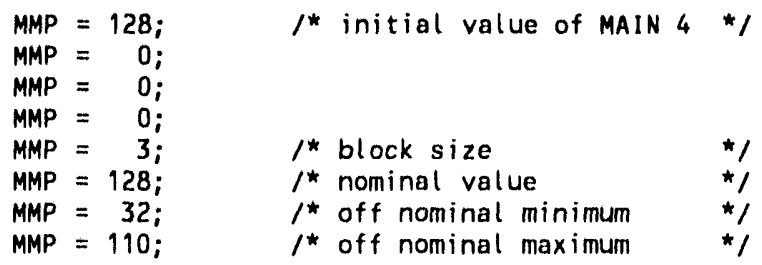

1 * now fill in the zeros representing the sub data */ for $(i=0 ; i<2 * 8 ; i++)$ MMP $=0$;

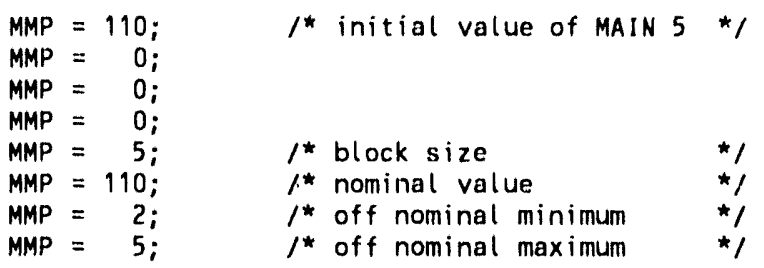

1 * now fill in the zeros representing the sub data */ for $(i=0 ; i<4 * 8 ; i++)$ MMP $=0$;

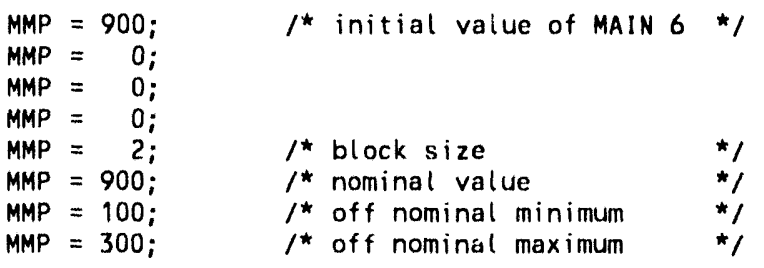

$l^{*}$ now fill in the zeros representing the sub data * $/$ for $(i=0 ; i<1 * 8 ; i++)$ MMP $=0$;

MMP $=75 ; \quad / *$ initial value of MAIN 7 */

MMP $=0$

MMP $=0$

MMP $=0 ;$

MMP $=1 ;$

MMP $=75 ;$

$M M P=45$

MMP $=60 ;$

$1^{*}$ block size

$l^{*}$ nominal value

$l^{*}$ off nominal minimum

$\star$

$l^{*}$ off nominal maximum

*/

$I^{*}$ now fill in the zeros representing the sub data * $/$ for $(i=0 ; i<0 * 8 ; i++)$ MMP $=0$;

MMP $=10 ; \quad l^{*}$ initial value of MAIN $8 * /$

MMP $=0 ;$

MMP $=0 ;$

MMP $=0 ;$

MMP $=1 ;$

MMP $=10 ;$

MMP $=5$

MMP $=10 ;$

/* block size

$l^{*}$ nominal value

$l^{*}$ off nominal minimum

$\star 1$
$\star 1$
$\star 1$
$\star 1$

$I^{*}$ now fill in the zeros representing the sub data * for $(i=0 ; i<0 * 8 ; i++)$ MMP $=0$; 


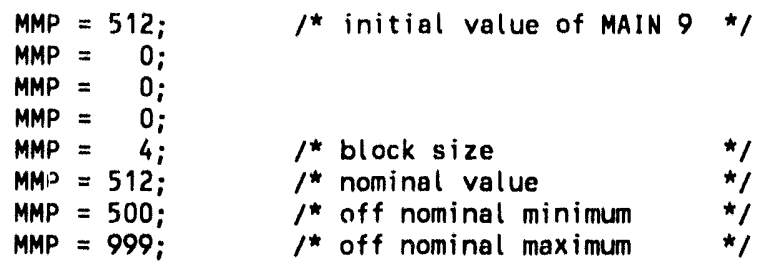

$I^{*}$ now fill in the zeros representing the sub data *

for $(i=0 ; i<3 * 8 ; i++)$ MMP $=0$;

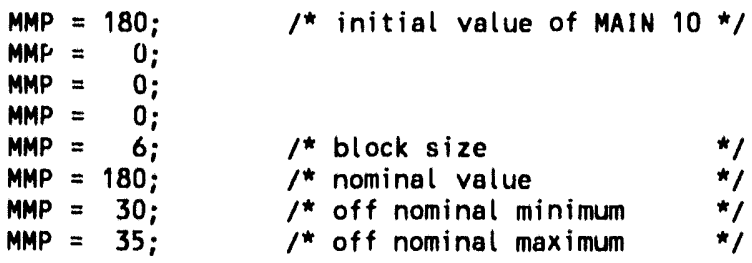

$I^{*}$ now fill in the zeros representing the sub data * for $(i=0 ; i<5 * 8 ; i++)$ MMP $=0$;

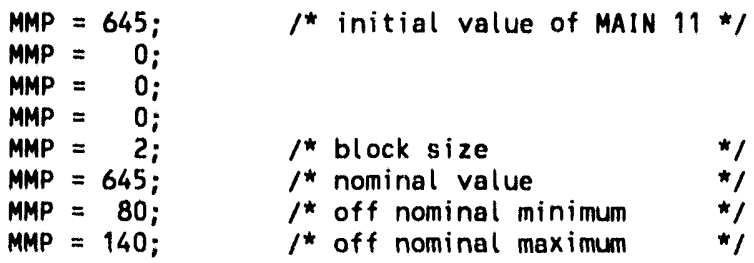

$I^{*}$ now fill in the zeros representing the sub data */ for $(i=0 ; i<1 * 8 ; i++)$ MMP $=0$;

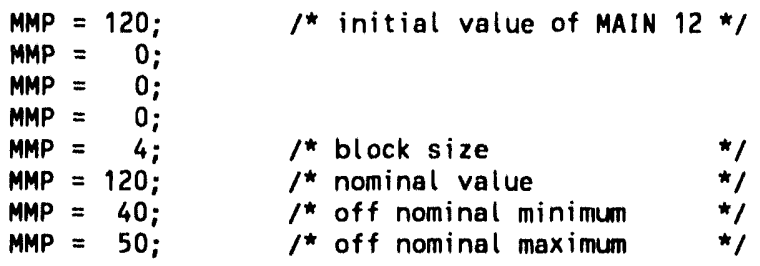

$I^{*}$ now fill in the zeros representing the sub data */ for $(i=0 ; i<3 * 8 ; i++)$ MMP $=0$;

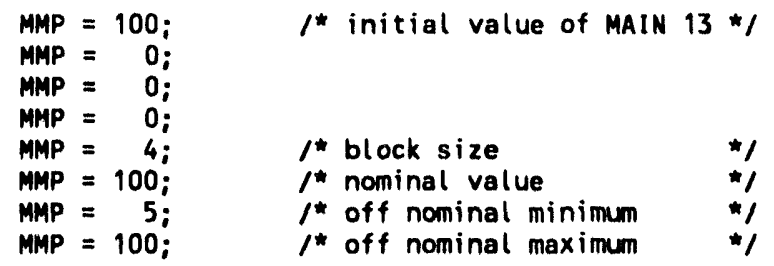

1 " now fill in the zeros representing the sub ciata */ for $(i=0 ; i<3 * 8 ; i++)$ MMP $=0$;

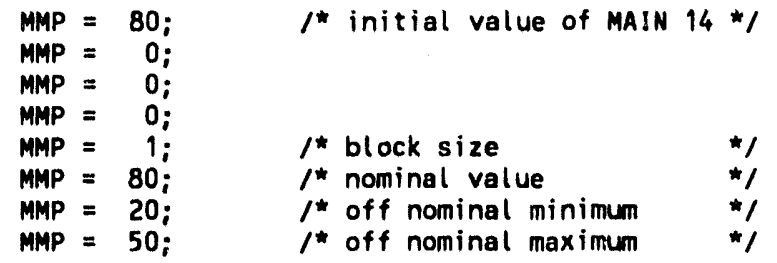




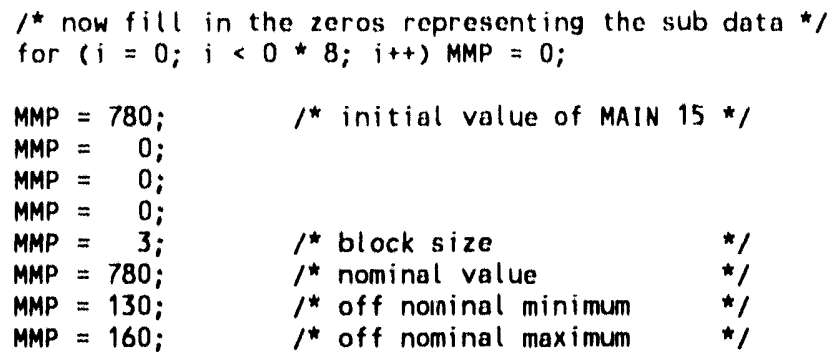

/* now fill in the zeros representing the sub data */ for $(i=0 ; i<2 * 8 ; i++)$ MMP $=0$;

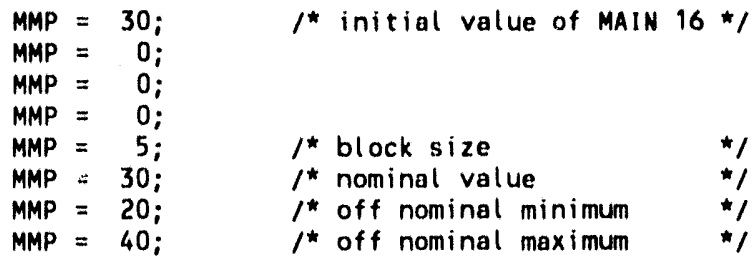

/* now fill in the zeros representing the sub data */ for $(i=0 ; i<4 * 8 ; i++) \operatorname{MMP}=0$;

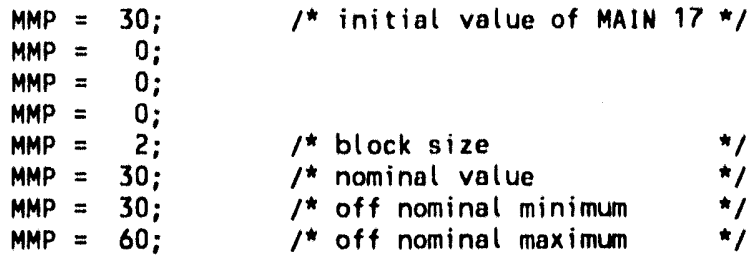

1 * now fill in the zeros representing the sub data */ for $(i=0 ; i<1 * 8 ; i++)$ MMP $=0$;

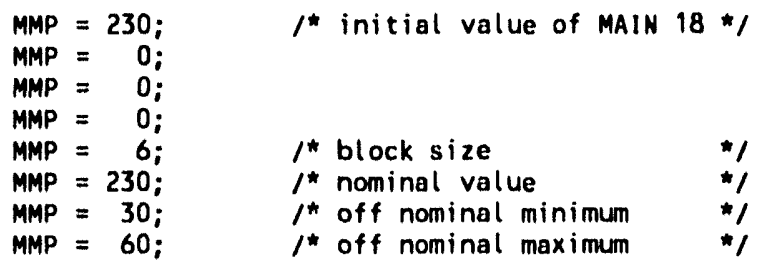

1 now fill in the zeros representing the sub data */ for $(i=0 ; i<5 * 8 ; i++)$ MMP $=0$;

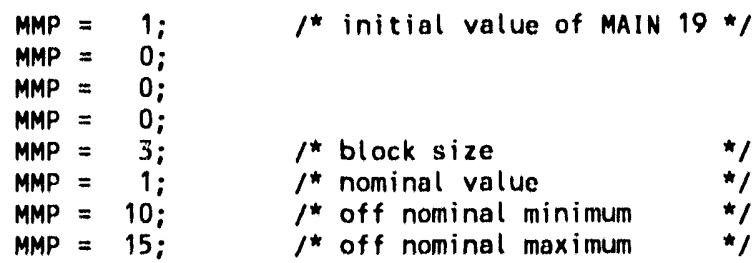

$I^{*}$ now fill in the zeros representing the sub data */ for $(i=0 ; i<2 * 8 ; i++)$ MMP $=0$;

MMP $=1022 ; \quad I^{*}$ initial value of MAIN $20 * /$

MMP $=0 ;$

MMP $=0 ;$

MMP $=0$ 


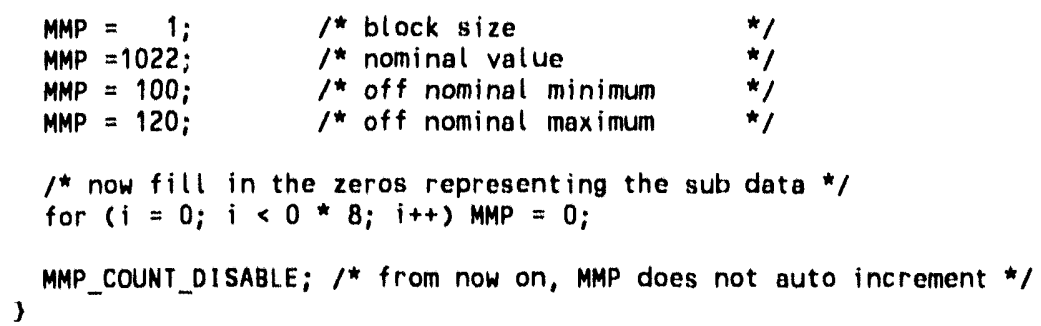




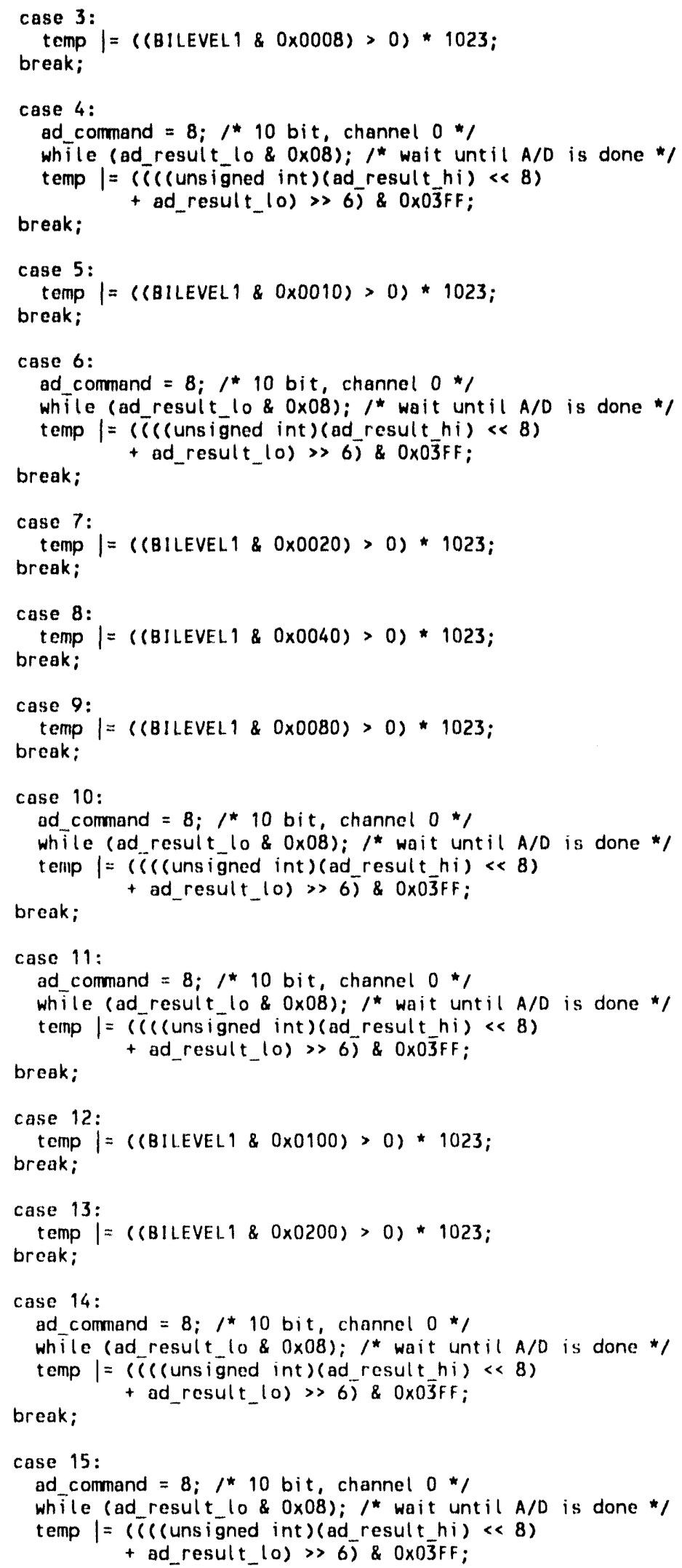




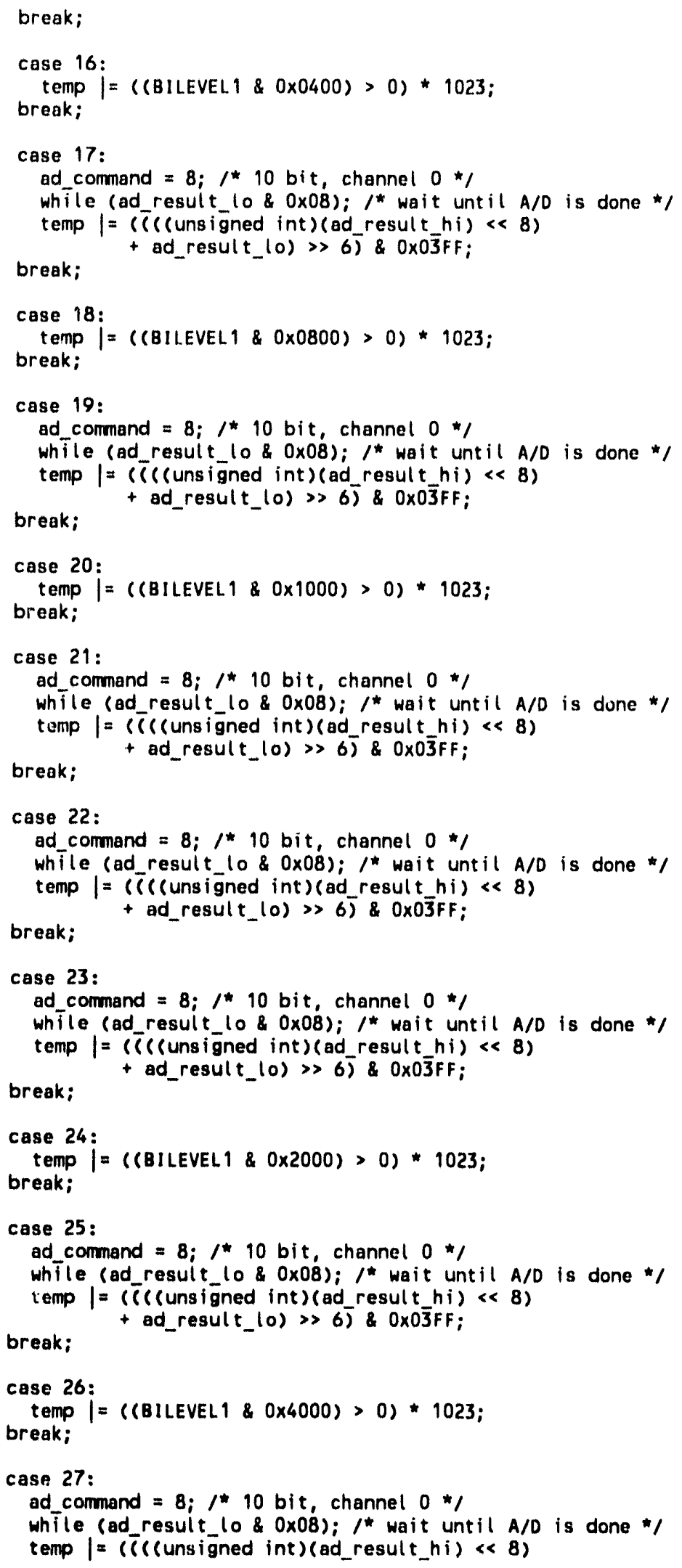




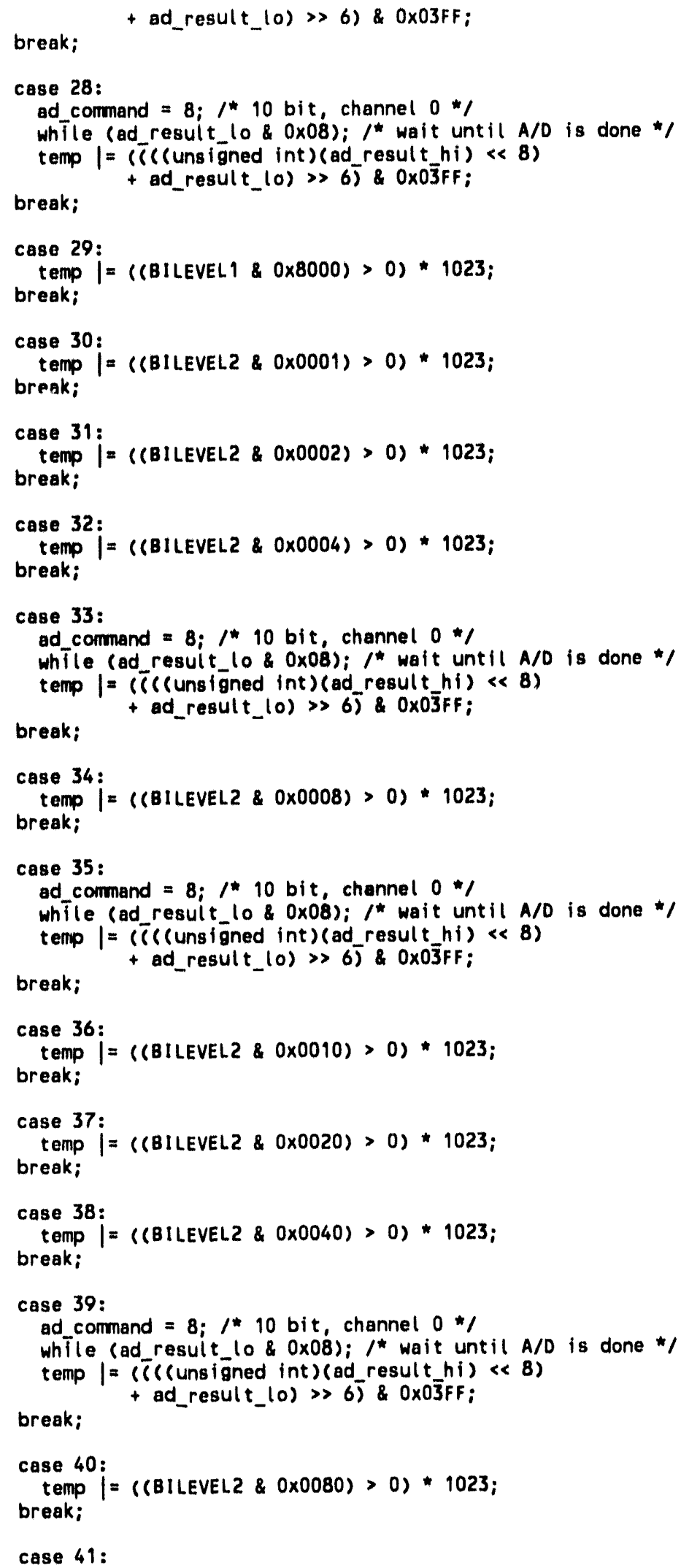




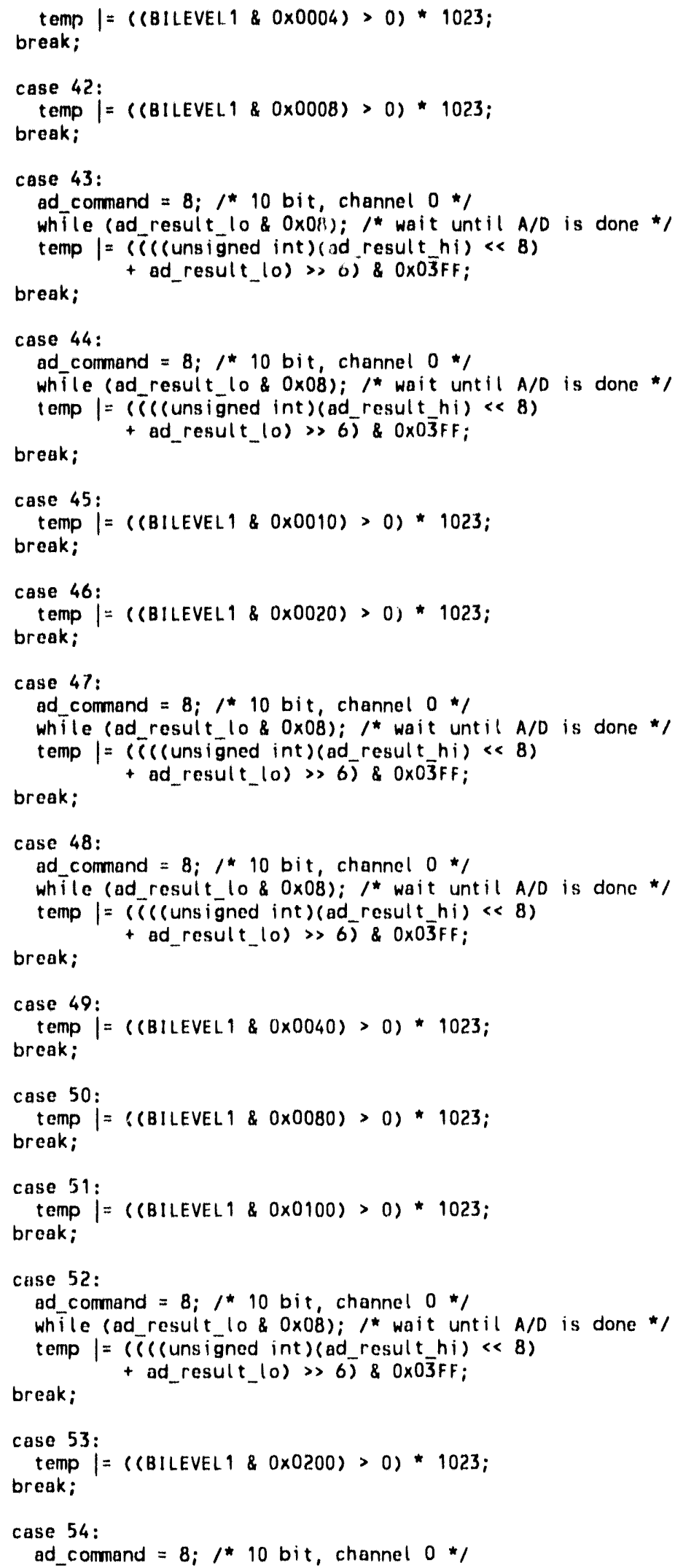




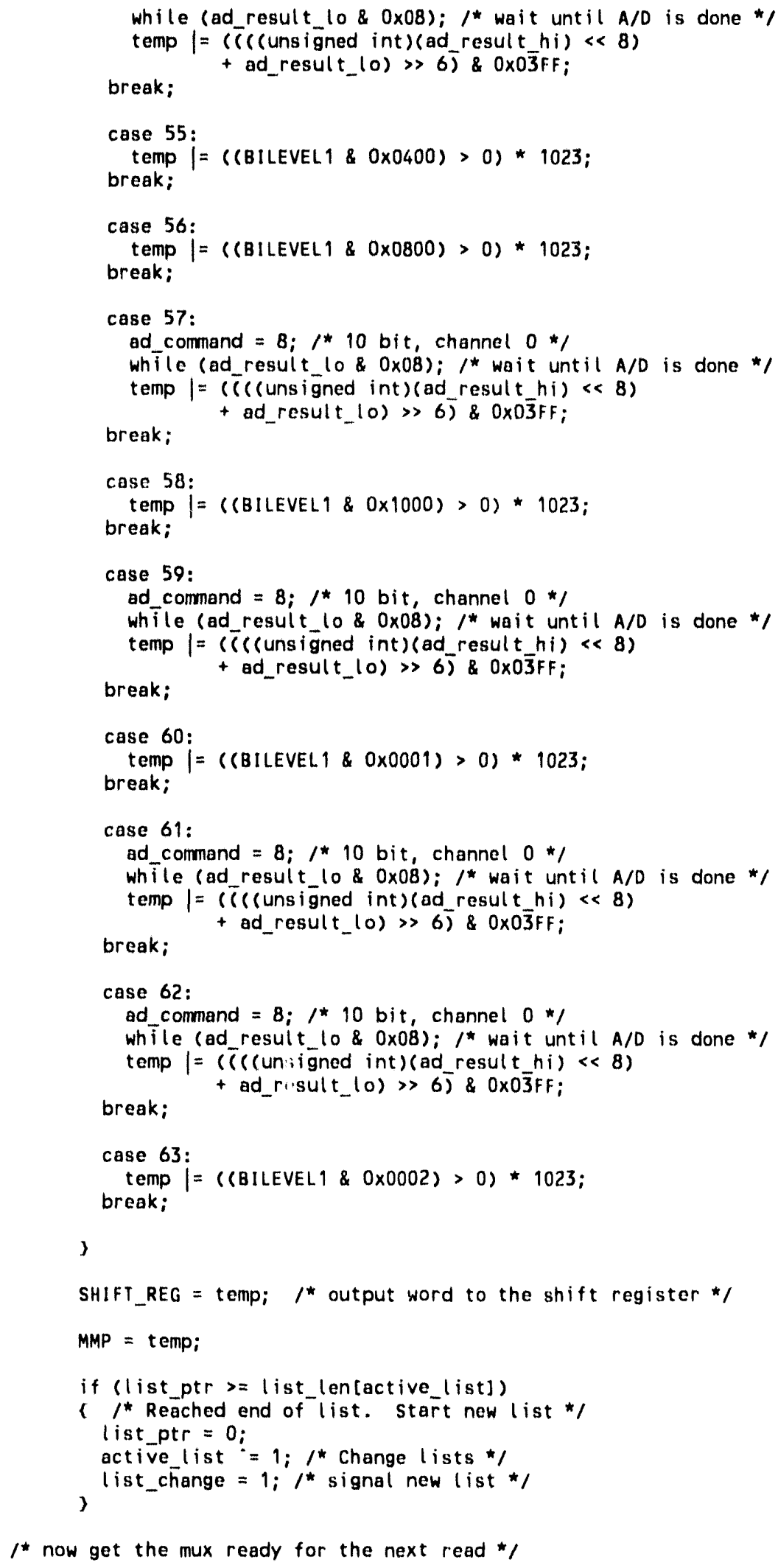




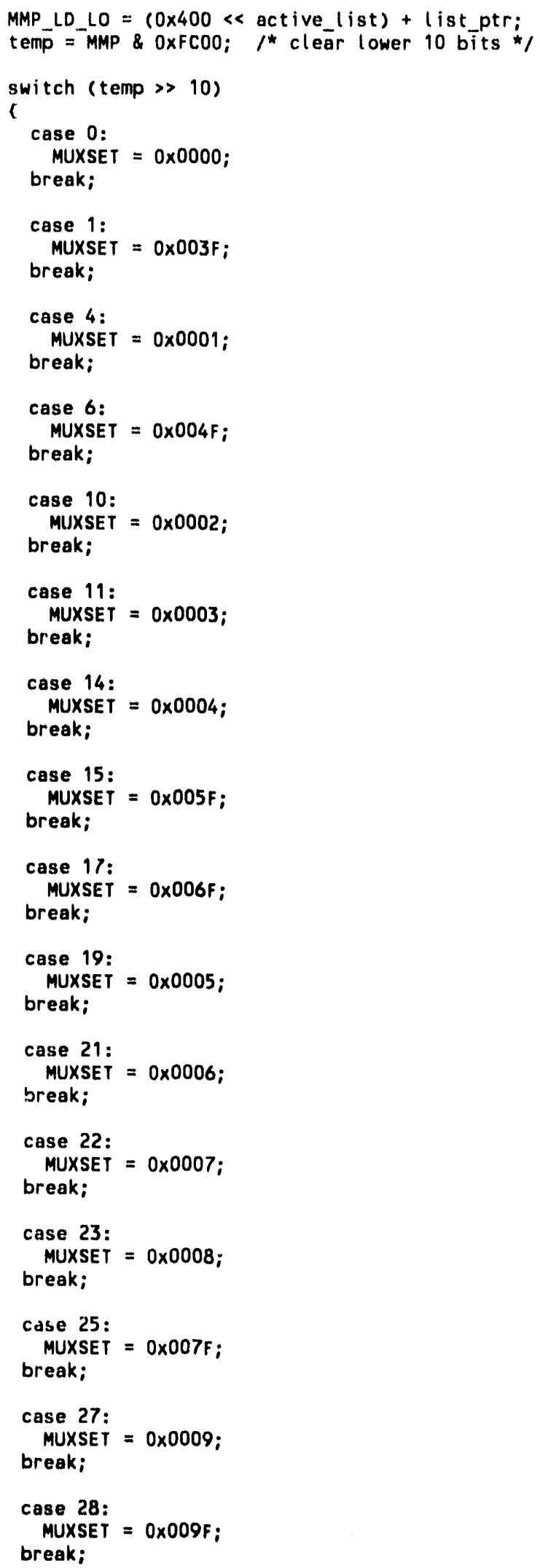




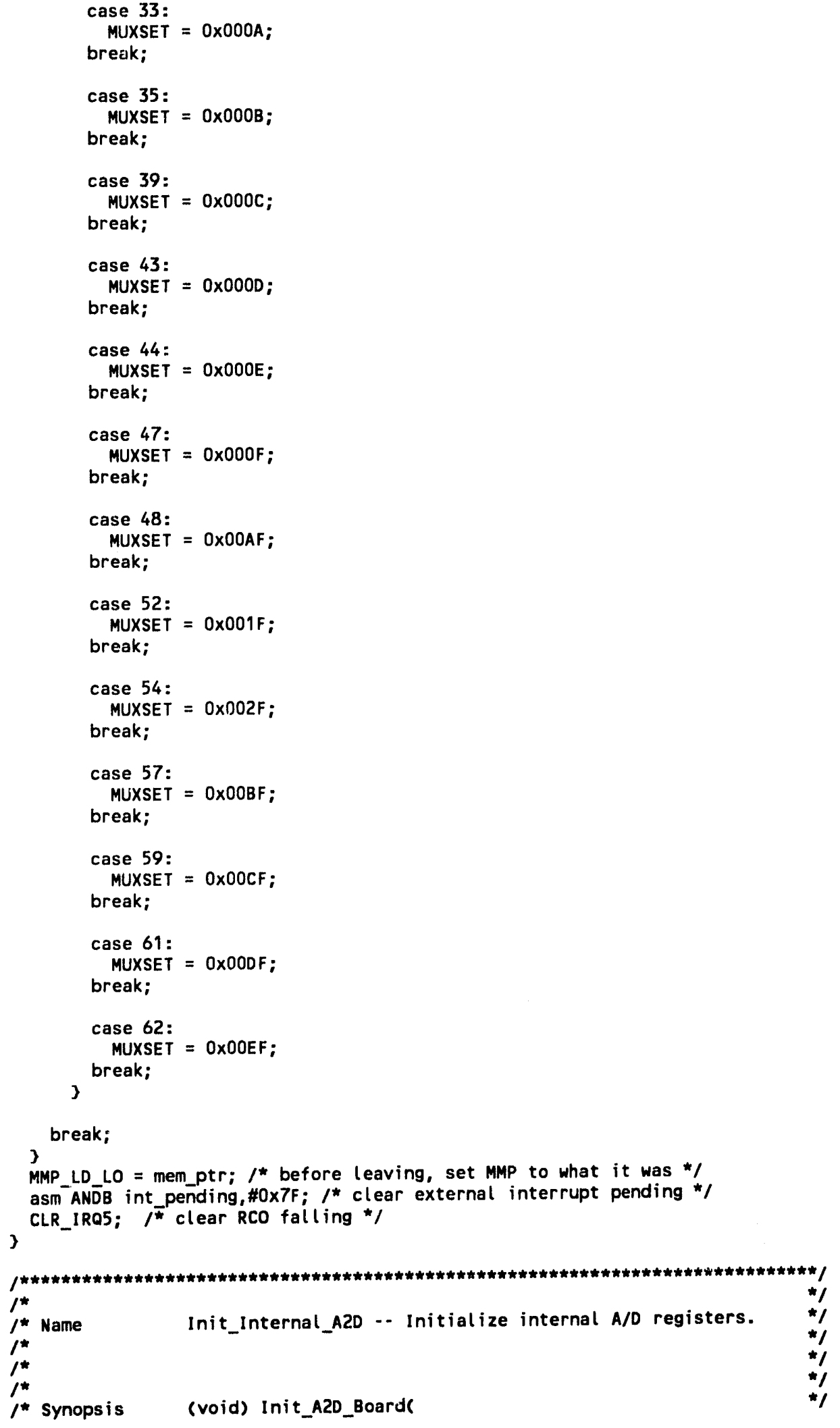




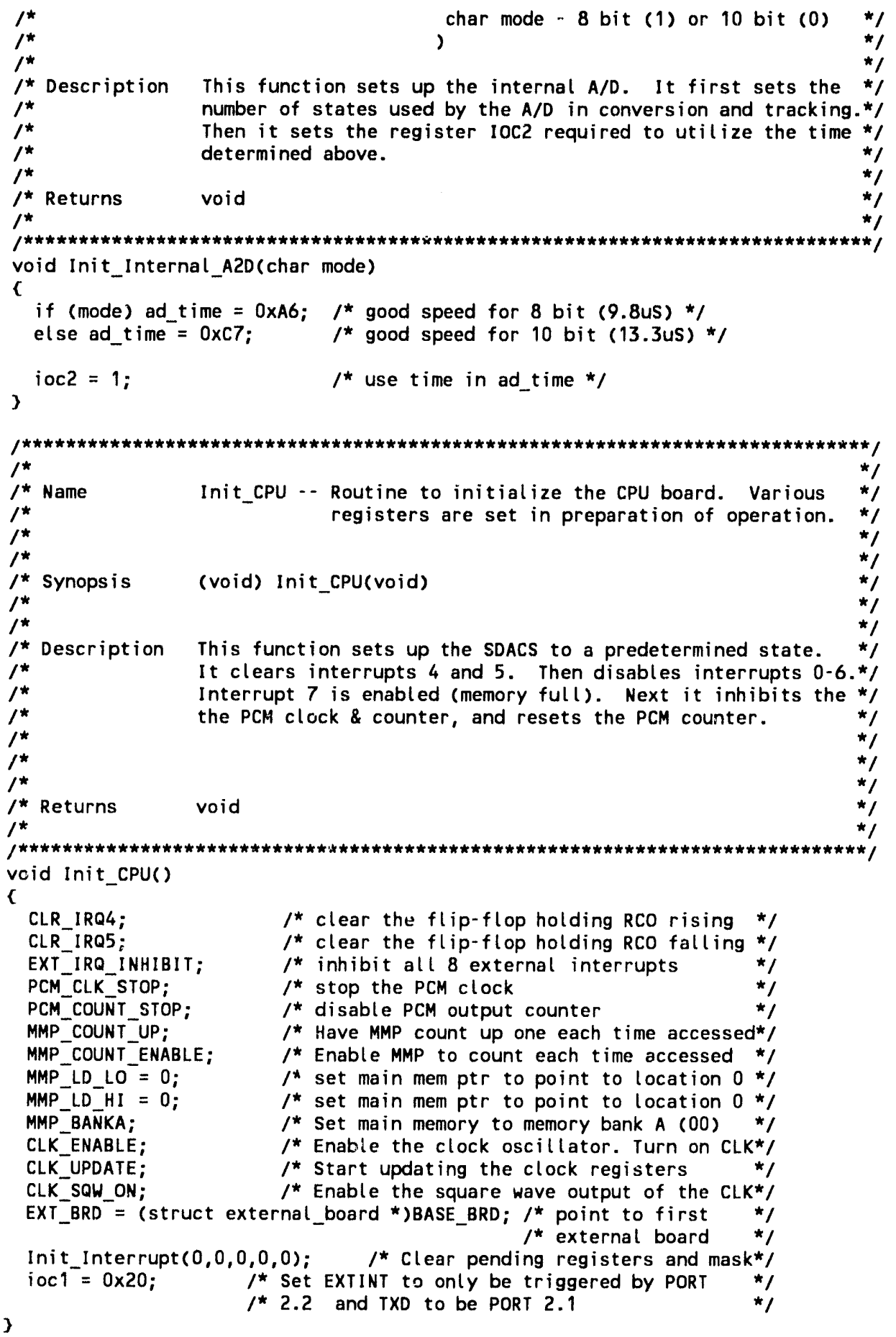

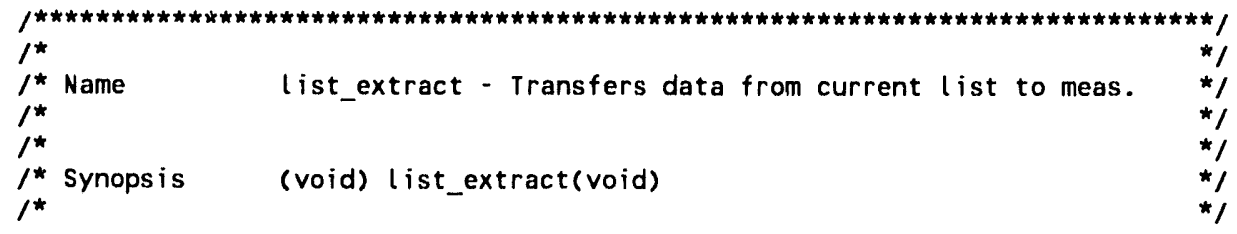




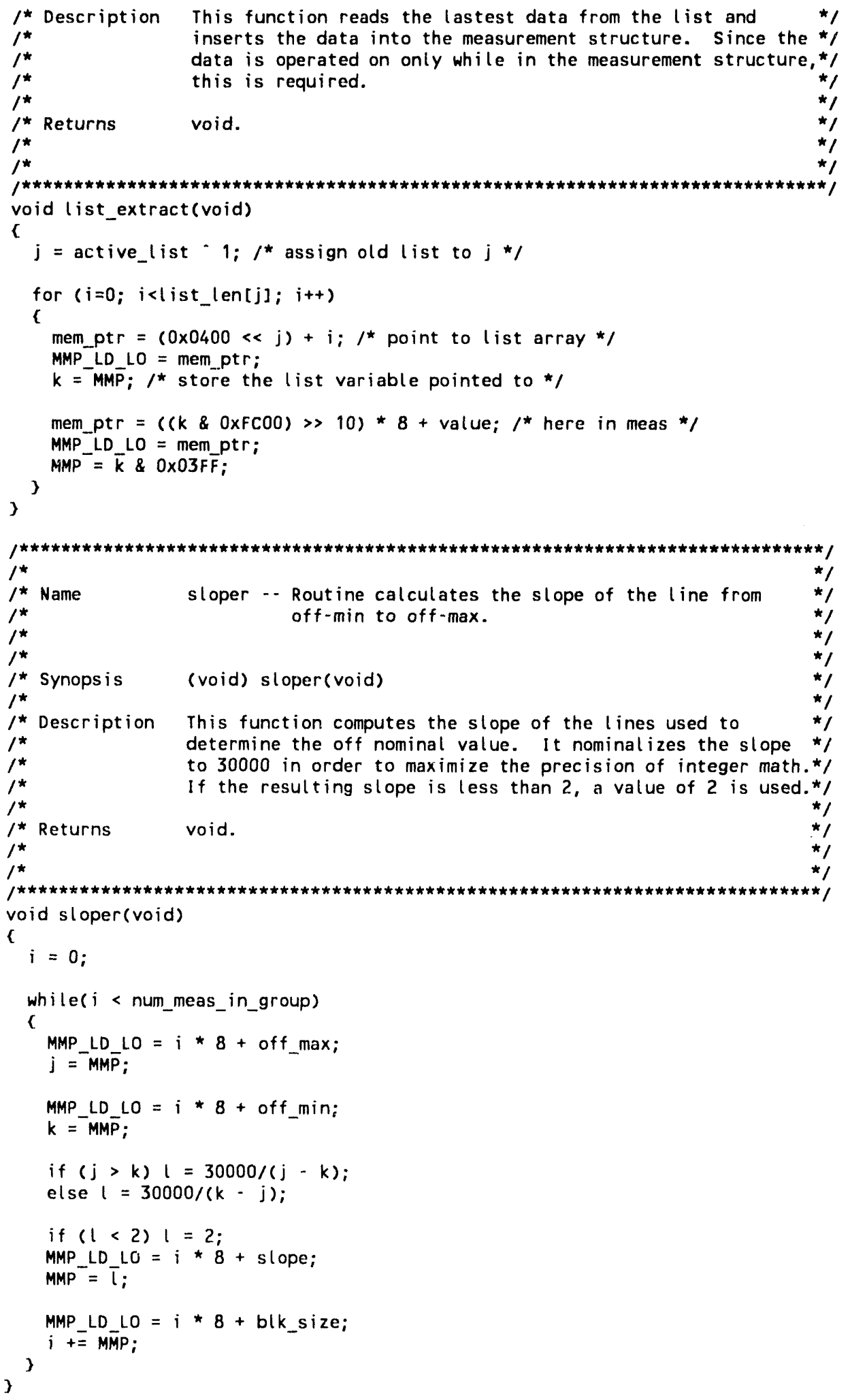




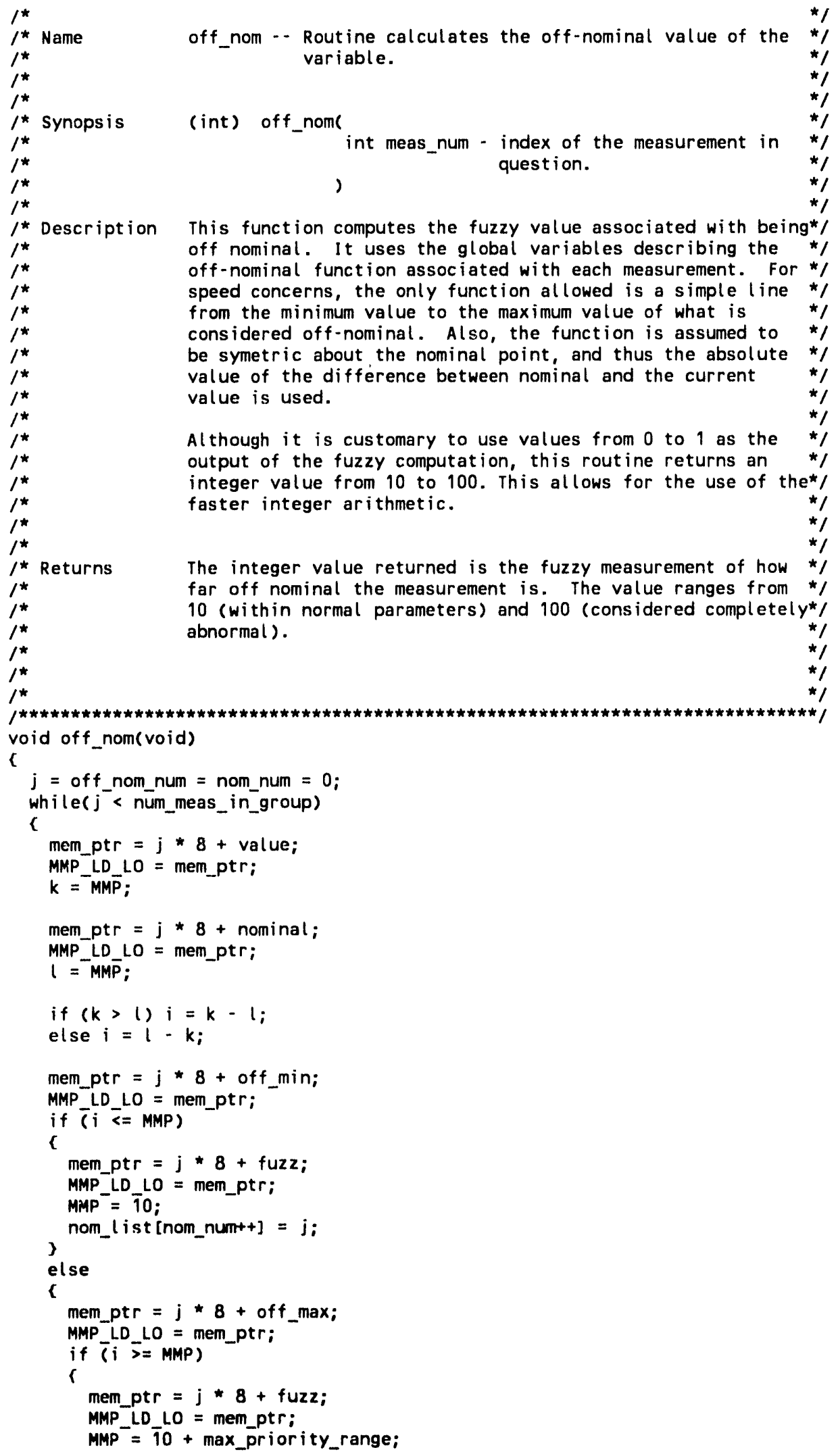




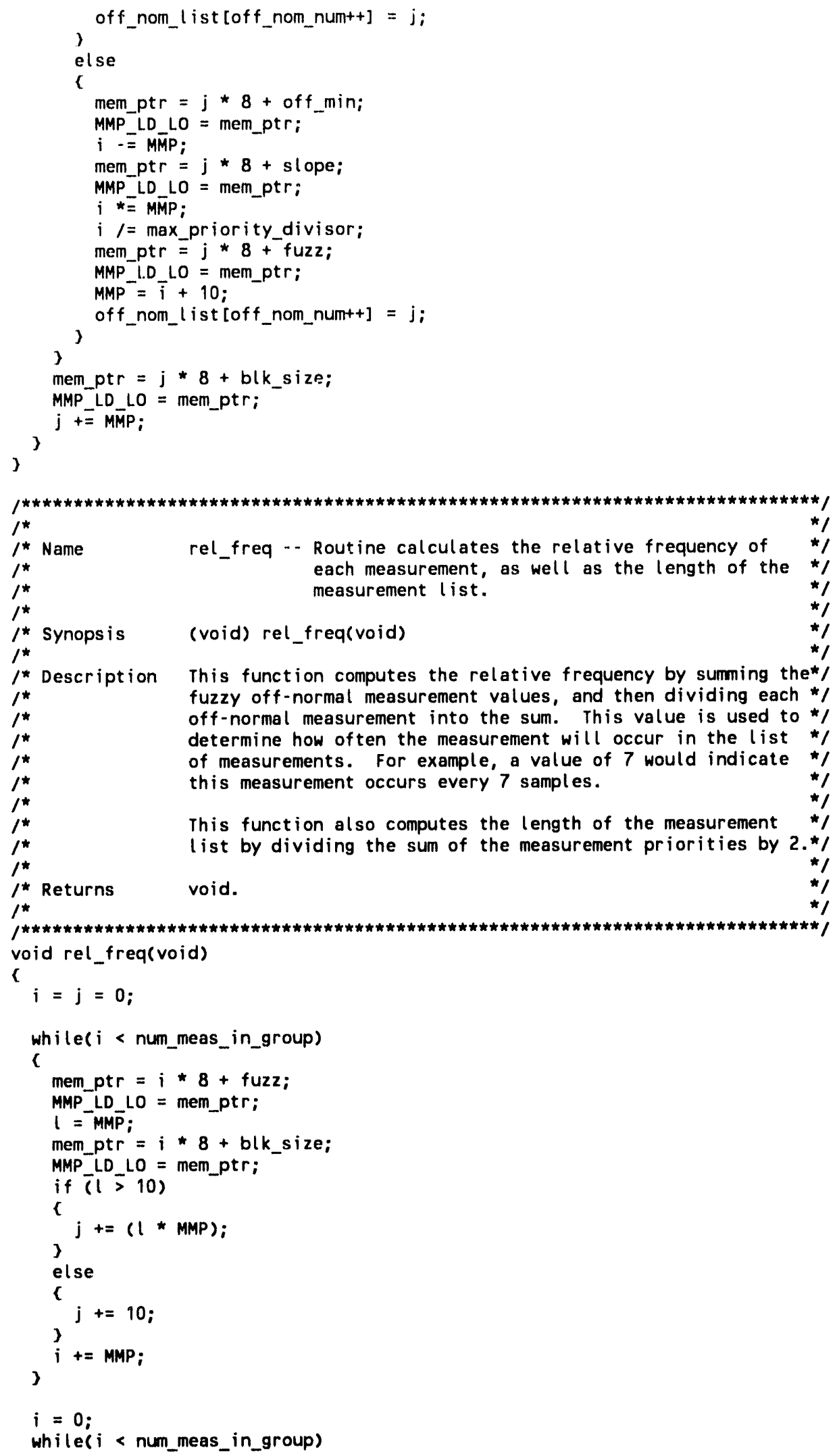




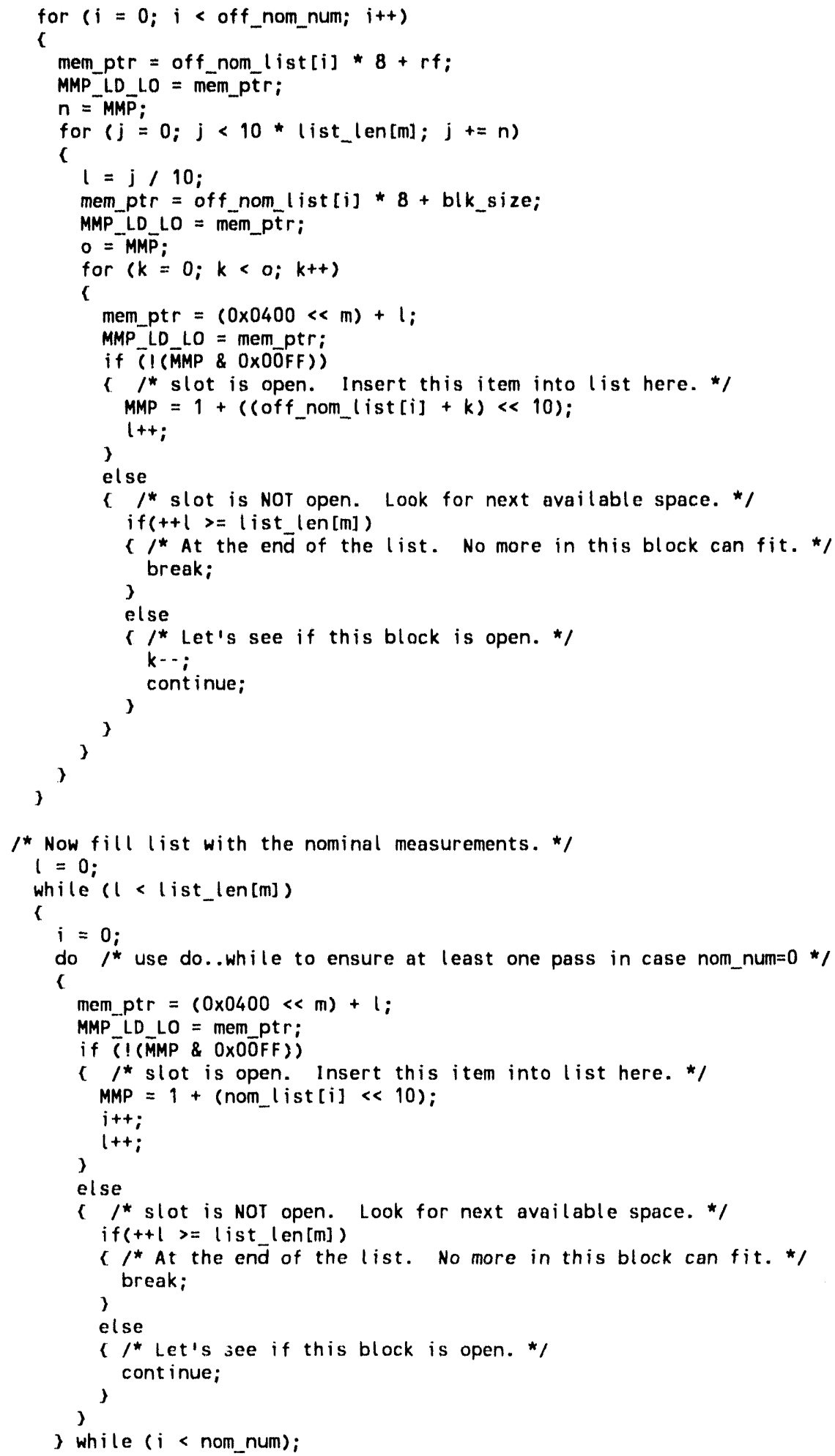




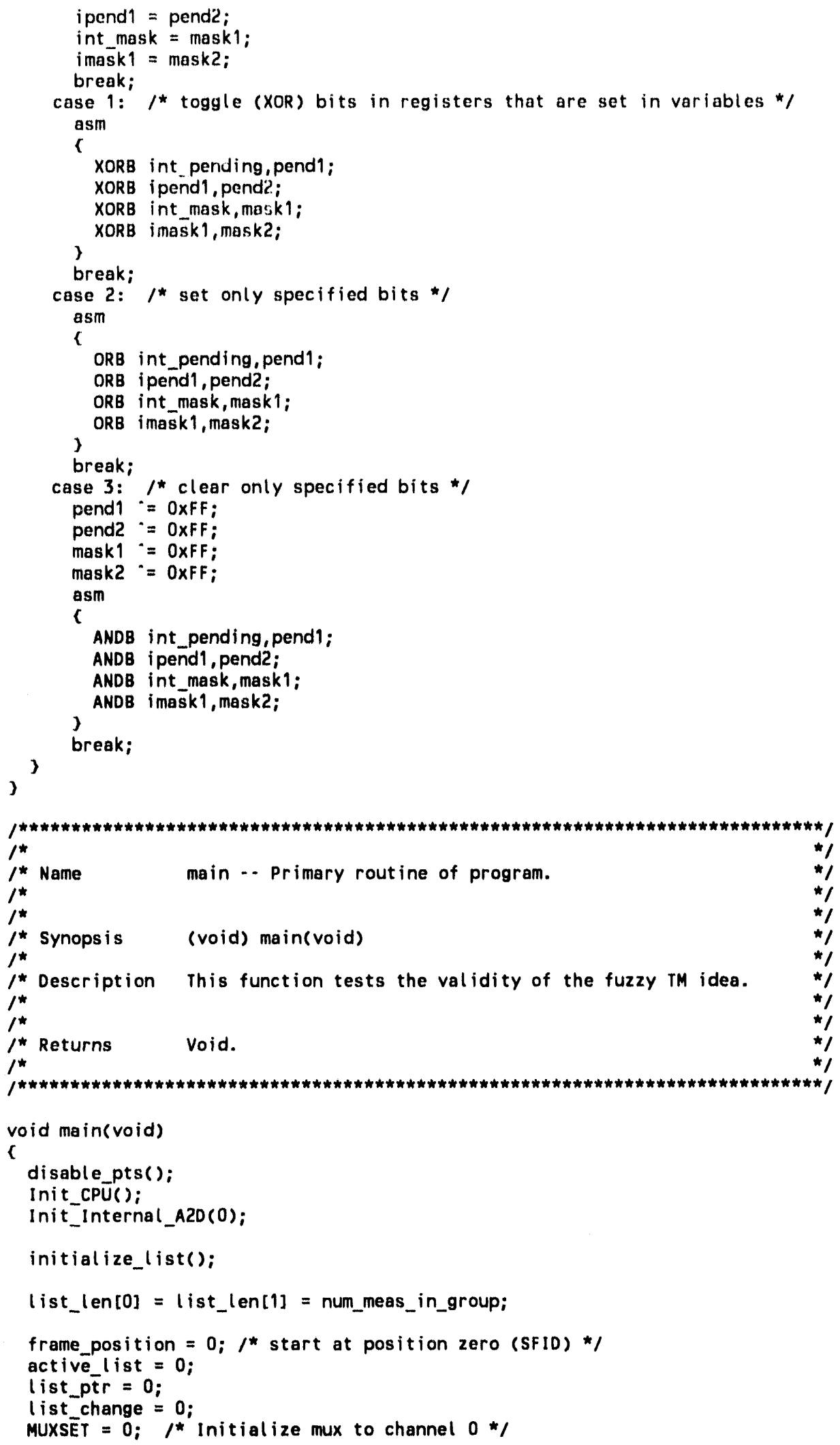


for $(i=0 ; 1<1$ ist_len $[0] ; i++)$

(

MMP_LD_LO $=\mathfrak{i} * 8+$ value;

$j=(i<10)+M M P$;

MMP_LD_LO $=0 \times 0400+i$

MMP $^{-}=\mathrm{I}_{\mathrm{i}}$

MMP LD $L O=0 \times 0800+i ;$

MMP'= $=\vec{j} ;$

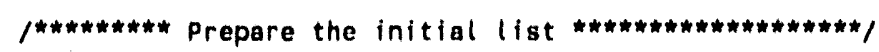

sloper():

list_extract();

off nom( ):

rel_freq();

stufff_list():

enable();

Init Interrupt $(2,0,0,0 \times 80,0) ; l^{*}$ enable only the external interrupt */

init_PCM();

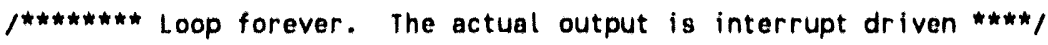

while $(1==1)$

r

if (list change)

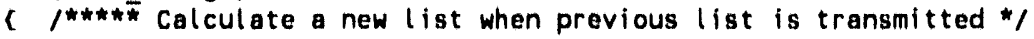

list_extract();

off nom();

rel freq():

stuff_list();

list_change $=0$;

$>$ 


\section{Appendix C}

The following software listing is of the program used to decommutate the data from the adaptive TM. 


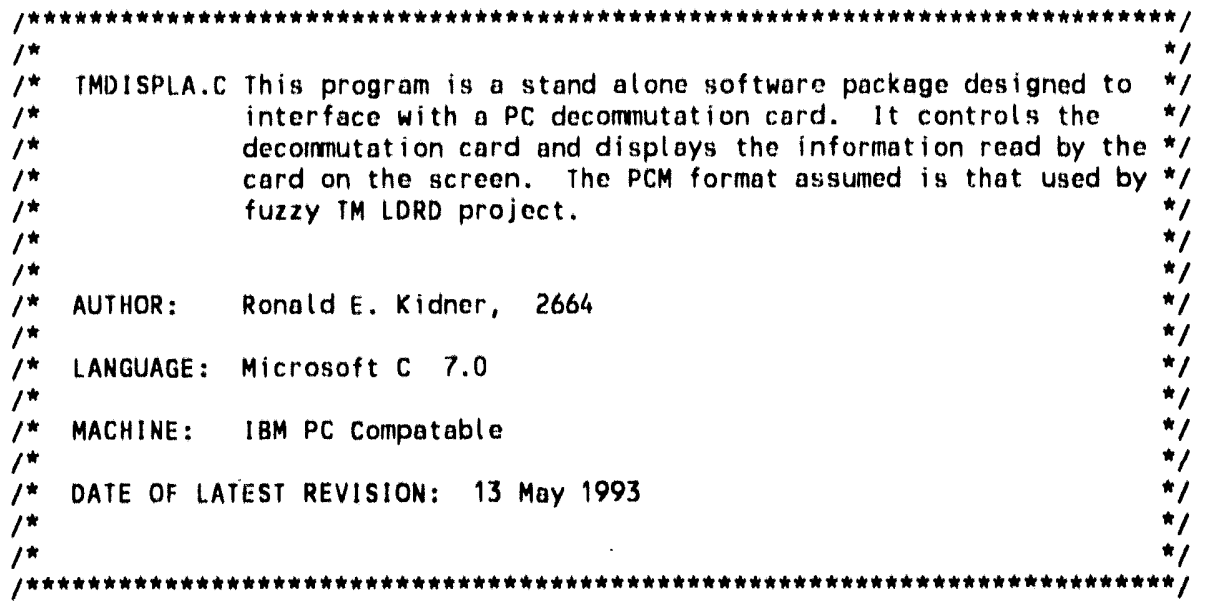

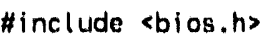

\#include 〈dos.h>

\#include sdecl.h $h$ >

\#include 〈conio.h〉

\#include 〈stdio.h>

\#include <stdlib.h>

\#include 〈t ime. $h\rangle$

\#include 〈graph.h>

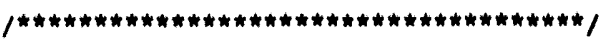

$f^{*} \quad$ Internal Functions

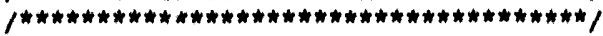

void updatescn (void);

interrupt far handler(void);

void debrief(void);

int readkbd(void);

void init(void);

void test intr(void);

uns igned char test, testie;

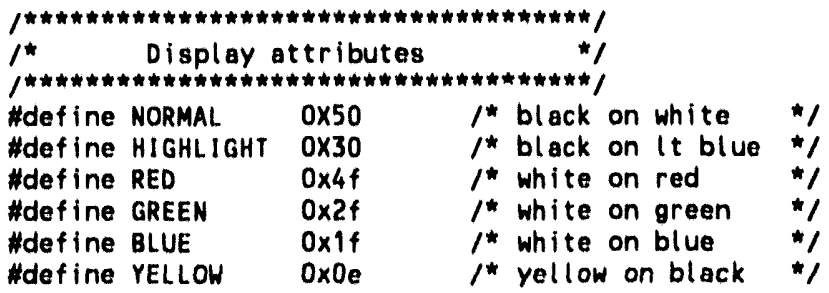

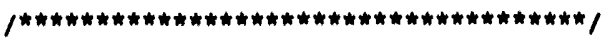

$1^{\star}$ Box, Line attributes */

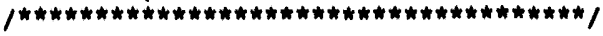

\#define HORIZ 1

\#define VERT 0

\#define SINGLE 0

\#define DOUBLE 15

$\begin{array}{ll}\text { \#def ine TRUE } & 1 \\ \text { \#def ine FALSE } & 0\end{array}$

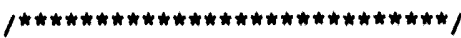

/* decom board addresses $\star /$

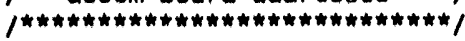

\#define pgm mode $0 \times 81$

\#define pgm_8255 (char *)0xd000c003

\#define pgm 1 Oxfa $/$ " lower 8 bits of counter load *

\#define pgm_2 $0 \times 47 \quad 1^{*}$ upper 3 bits of counter load *

\#define pgm_3 $0 \times 40$ 


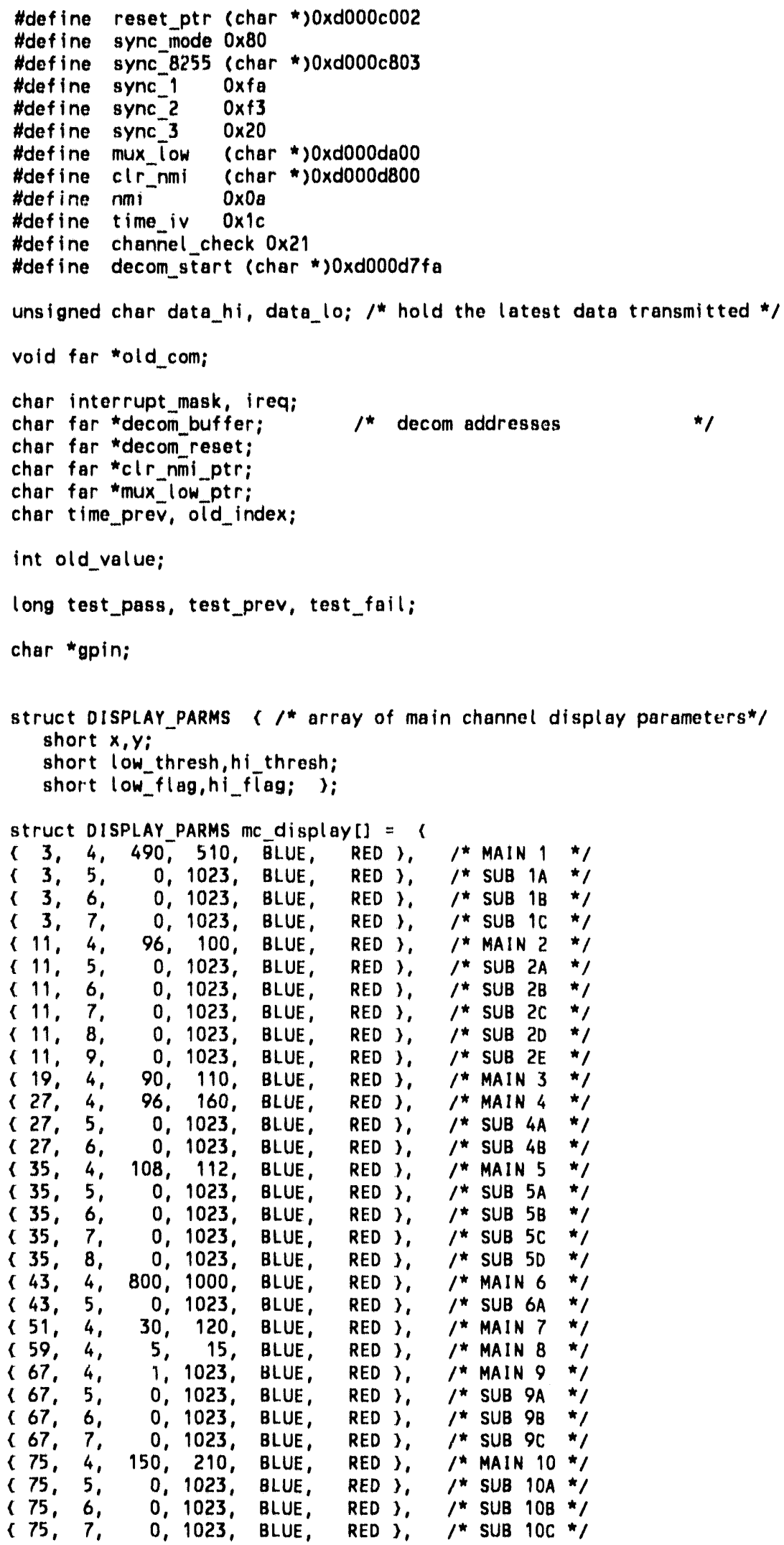




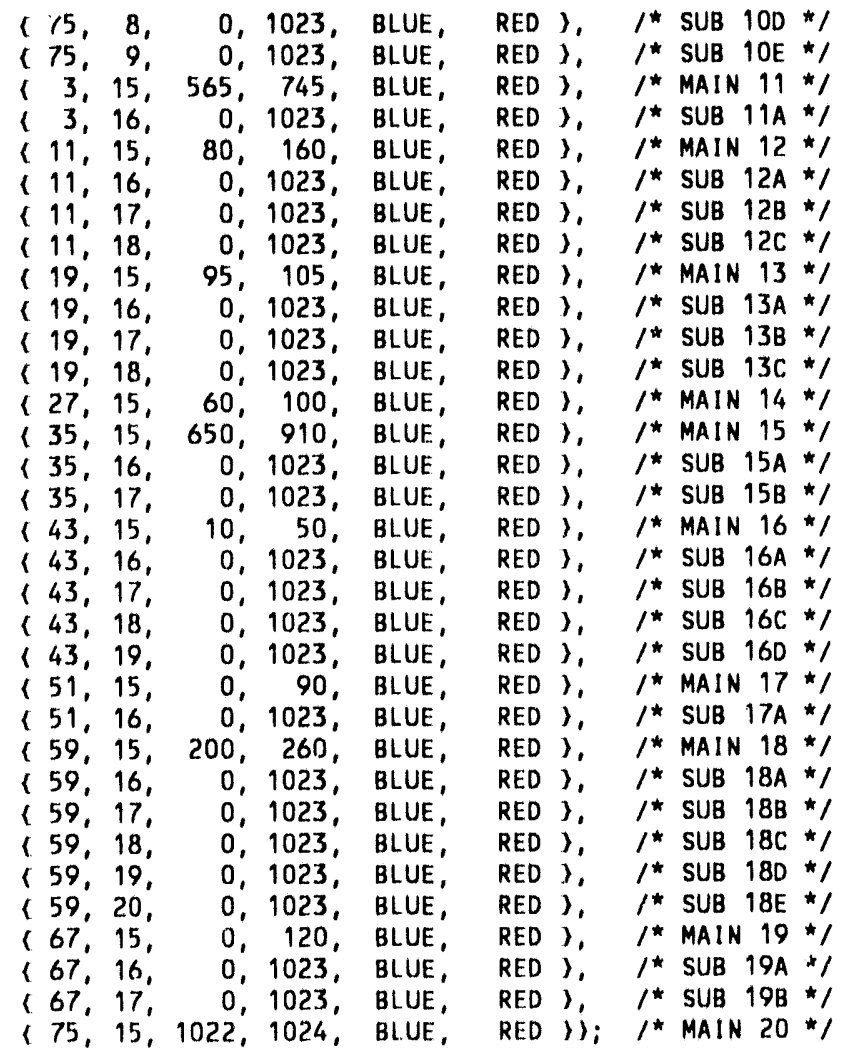

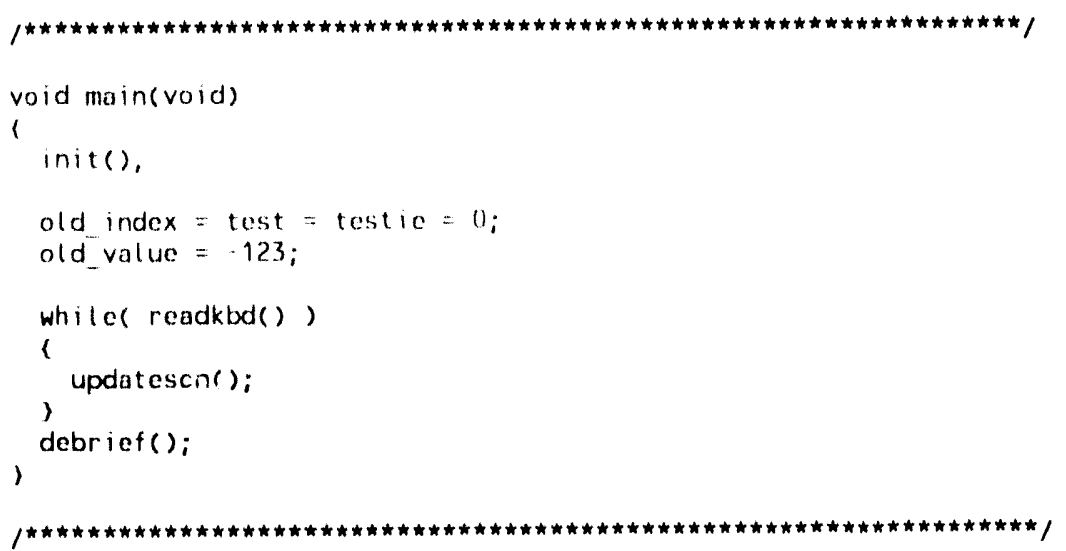




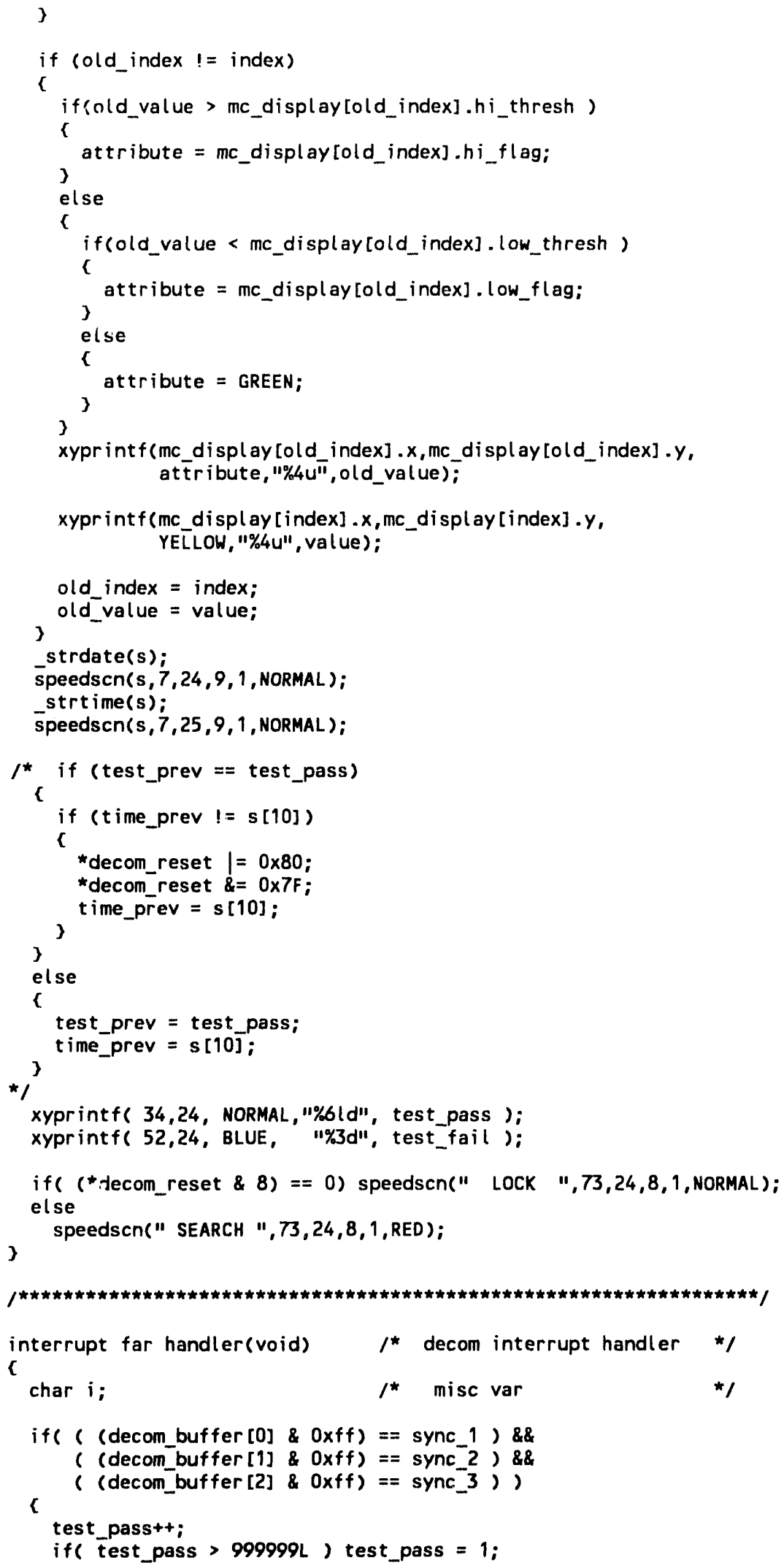

ifs ( (decom_buffer [0] \& $0 x f f)==$ sync_1 ) \&\&

( (decombuffer [1] \& Oxff) $==$ sync 2 ) \&\&

c 
(inescn $(16,11,9, \operatorname{siNGLE}, 1,1$, NORMAL, HORI 2$)$; ( inescn $(24,11,9$, SINGLE , 1, 1 , NORMAL, HORIZ); ( inescn $(32,11,9$, SINGLE 1, 1, NORMAL, HOR I2); ( inescn $(40,11,2, \operatorname{sinGLE}, 1,1$, NORMAL, HOR I 2$)$; l inescn $(48,11,9, \operatorname{siNGLE}, 1,1$, NORMAL, HOR I 2$)$; (inescn(56,11,9, SINGLE, 1, 1, NORMAL, HORIZ); ( inescn $(64,11,9, \operatorname{siNGLE}, 1$, 1 , NORMAL, HORI2); ( inescn $(72,11,9$, SINGLE, 1,2, NORMAL, HORI 2$)$; (inescn( $1,12,8$, SINGLE , 2, 1, NORMAL, HORIZ); (inescn $88,12,9$, SINGLE, 1, 1, NORMAL, HOR IZ);

I inescn $(16,12,9$, SINGLE 1 1, 1, NORMAL, HORIZ); (inescn(24,12,9, SINGLE, 1, 1, NORMAL, HORIZ); ( inescn (32, 12,9, SINGLE, 1, 1, NORMAL, HORIZ); ( inescn $(40,12,9$, SINGLE, 1,1 , NORMAL, HORIZ); ( inescn $(48,12,9$, SINGLE , 1, 1, NORMAL, HORIZ); ( inescn $(56,12,9$, SINGLE, 1, 1, NORMAL, HORIZ); I inescn $(64,12,9$, SINGLE , 1, 1, NORMAL, HORIZ); I inesen( $72,12,9$, SINGLE, 1,2, NORMAL, HORIZ);

speedscn("GRP $9 ", 2,2,5,1$, HIGHLIGHT); speedscn("GRP 2"10,2,5,1, HIGHLIGHT); speedscn("GRP 3", 18,2,5,1, HIGHLIGHT); speedsen("GRP 4", 26, 2,5, 1, HIGHLIGHT); speedsen ("GRP 5", 34, 2,5,1, HIGHLIGHT); speedscn ("GRP 6", 42,2,5,1, H IGHL IGHT); speedscn("GRP 7",50,2,5,1, HIGHLIGHT); speedsen("GRP 8", 58,2,5, 1, HIGHLIGHT); speedsen ("GRP 9",66, 2,5,1, HIGHLIGHT); speedsen ("GRP 10", 74, 2,6,1, HIGHLIGHT); speedscn("GRP 11",2, 13,6,1, HIGHLIGHT) speedscn("GRP 12", 10, 13,6, 1, HIGHLIGHT); speedscn("GRP 13", 18, 13,6,1, HIGHLIGHT); speedscn("GRP 14", 26, 13,6,1, HIGHLIGHT); speedscn("GRP 15",34,13,6,1, HIGHLIGHT); speedscn("GRP 16", 42, 13,6,1, HIGHLIGHT); speedscn("GRP 17",50,13,6,1, HIGHLIGHT); speedscn("GRP 18",58, 13,6,1, HIGHLIGHT); speedscn("GRP 19",66, 13,6, 1, HIGHLIGHT); speedscn("GRP 20",74,13,6,1, HIGHLIGHT);

speedscn("Adapt ive Telemetry PCM Decomutat ion",2, 23, 35, 1, NORMAL); speedsen ("Decom Status:", 59, 24,13,1, NORMAL);

speedscn ("F1-EXIT", 18, 25,7,1, NORMAL);

speedscn("F 2-RESET", 27, 25,8, 1, NORMAL);

speedscn("Frames: Val id $=" 1,18,24,16,1$, NORMAL);

speedscn("Inval id $=", 42,24,10,1$, NORMAL);

speedsen ("

speedsen $"$ "

$", 16,23,37,1$, NORMAL );

strdate(s):

speedsen("Date:", 2,24,5, 1, NORMAL);

speedscn( $5,7,24,9,1$, NORMAL);

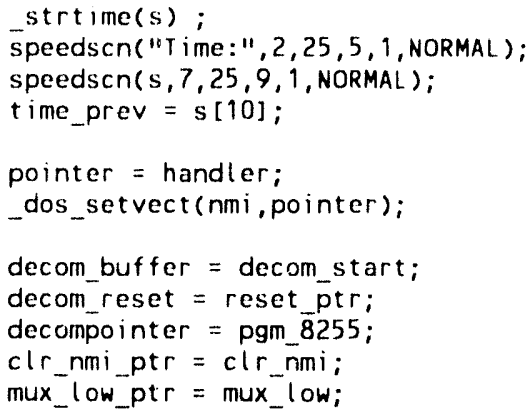

/* set decom IV */

$\begin{array}{lll}/ * & \text { set buffer pointer } & * / \\ / * & \text { set reset pointer } & * / \\ / * & \text { set decom board } & * / \\ / * & \text { set } \operatorname{cl} \text { nmi ptr } & * /\end{array}$


*(decompointer-1) $=$ pgm 3

* $($ decompointer -2$)=$ pgm_2;

* $($ decompointer -3$)=$ pgm_1;

decompointer $=$ sync 8255 ;

*decompointer = sync_mode;

* (decompointer -1$)=$ sync 3 ;

* $($ decompointer -2$)=$ sync_2;

* $($ decompointer -3$)=$ sync_1;

$i={ }^{*}$ mux low ptr;

*decom_reset $1=0 \times 80$.

$I^{*}$ reset mux.. low ff

*

*decom reset \& $=0 \times 7 f$

$i={ }^{\star} c l r$ nmi_ptr;

$/{ }^{\star}{ }^{*}$ decompointer $=0 \times O E$;

$l^{*}$ send reset off

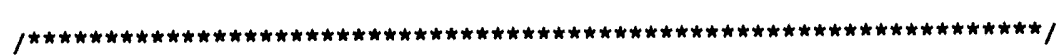




\section{DISTRIBUTION:}

$\begin{array}{lll}1 & 2600 & \text { J. H. Stichman } \\ 1 & 2664 & \text { D. E. Ryerson } \\ 1 & 2664 & \text { V. P. Salazar } \\ 10 & 2664 & \text { R. E. Kidner } \\ 1 & 9811 & \text { A. C. Wilken } \\ 1 & 8523-2 & \text { Central Technical Files } \\ 5 & 7141 & \text { Technical Library } \\ 1 & 7151 & \text { Technical Publications } \\ 10 & 7613-2 & \text { Document Processing for DOE/OSTI }\end{array}$



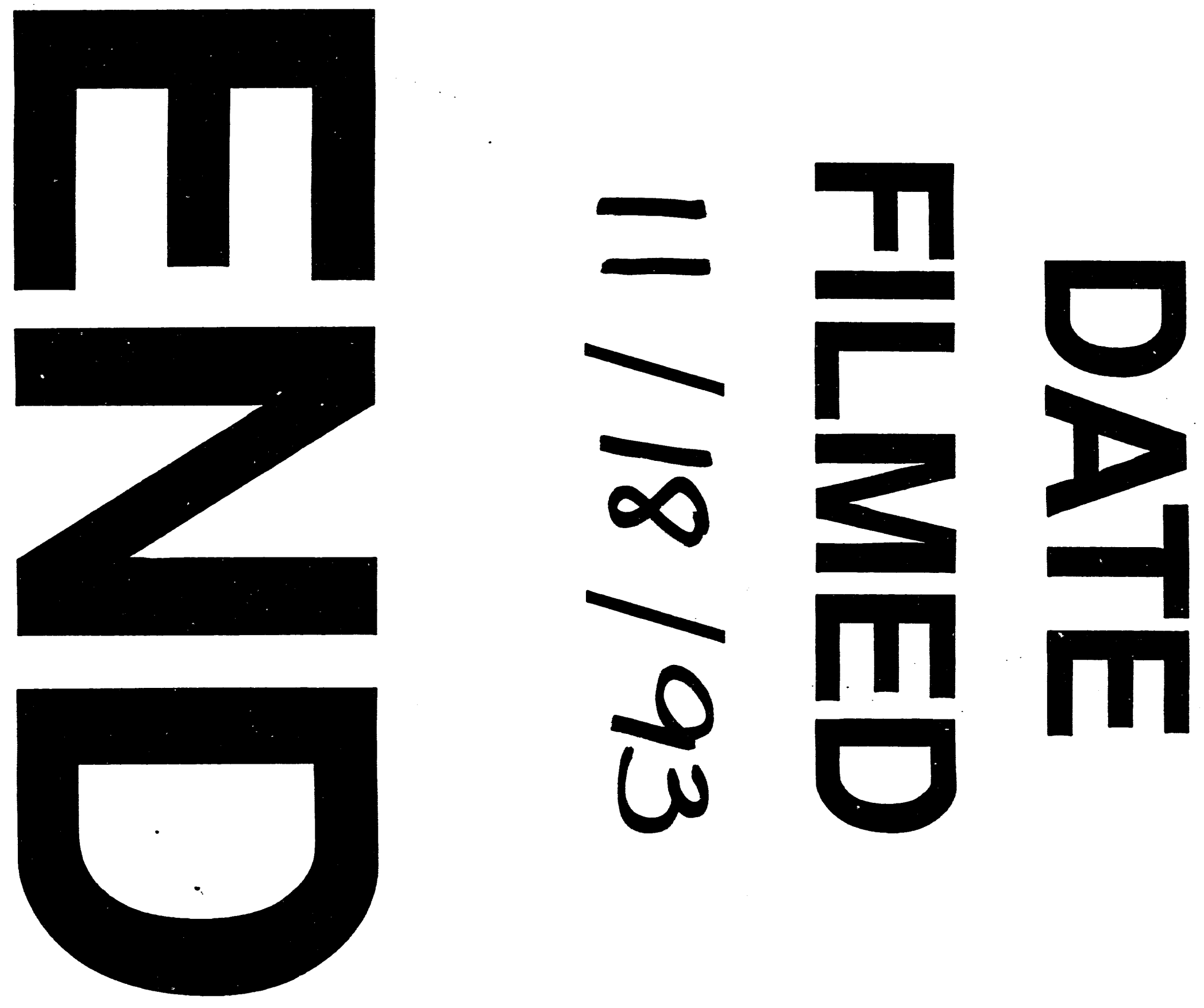
